\title{
D-branes in Landau-Ginzburg models and algebraic geometry
}

\section{Anton Kapustin}

California Institute of Technology, Department of Physics

Pasadena, CA 91125, U.S.A.

E-mail: kapustin@theory.caltech.edu

\section{Yi Li}

California Institute of Technology, Department of Physics

Pasadena, CA 91125, U.S.A.

E-mail: yili@theory.caltech.edu

Abstract: We study topological D-branes of type B in $N=2$ Landau-Ginzburg models, focusing on the case where all vacua have a mass gap. In general, tree-level topological string theory in the presence of topological D-branes is described mathematically in terms of a triangulated category. For example, it has been argued that B-branes for an $N=2$ sigmamodel with a Calabi-Yau target space are described by the derived category of coherent sheaves on this space. M. Kontsevich previously proposed a candidate category for Bbranes in $N=2$ Landau-Ginzburg models, and our computations confirm this proposal. We also give a heuristic physical derivation of the proposal. Assuming its validity, we can completely describe the category of B-branes in an arbitrary massive Landau-Ginzburg model in terms of modules over a Clifford algebra. Assuming in addition Homological Mirror Symmetry, our results enable one to compute the Fukaya category for a large class of Fano varieties. We also provide a (somewhat trivial) counter-example to the hypothesis that given a closed string background there is a unique set of D-branes consistent with it.

Keywords: Topological Field Theories, D-branes, Differential and Algebraic Geometry. 


\section{Contents}

1. Introduction 2

2. Mirror Symmetry for Fano varieties and LG models 5

3. General properties of B-branes in LG models 7

4. B-Branes in the LG model with $W=x y$

4.1 Preliminaries 9

4.2 Equations of motion and SUSY transformations 10

4.3 Spectrum of D0-D0 strings 11

4.4 Spectrum of D2-D2 strings 13

4.5 Spectrum of D0-D2 strings 15

5. Topological correlators in the LG model $W=x y \quad \mathbf{1 7}$

5.1 Topological B-twist 17

5.2 Boundary observables associated with the D0-brane 18

5.3 Boundary observables associated with the D2-brane 18

5.4 The boundary operator product algebra 18

6. B-branes in general massive LG models 21

6.1 Generalities 21

6.2 The LG model with the superpotential $W=x y+z^{2}$

6.3 The LG model with the superpotential $W=z_{1}^{2}+\cdots+z_{n}^{2}$

7. B-branes and twisted complexes 26

7.1 Kontsevich's proposal 26

7.2 A physical derivation of Kontsevich's proposal 27

7.3 Checking Kontsevich's proposal 29

7.4 B-branes, Clifford modules, and Koszul duality 32

8. Application: the category of A-branes for some Fano varieties 34

8.1 A-branes on $\mathbb{C P}^{2}$

8.2 A-branes on $\mathbb{C P}^{1} \times \mathbb{C P}^{1}$

9. Comments and outlook 38

A. Clifford algebras and modules 40 


\section{Introduction}

Topological open strings and topological D-branes have recently been enjoying the attention of both physicists and mathematicians. The most obvious physical motivation for studying topological string theory is that it is a toy-model for "physical" string theory. Thus a better understanding of topological D-branes could shed light on the general definition of a boundary condition for a two-dimensional conformal field theory (2d CFT), something which is not known at present. Further, if a $2 \mathrm{~d}$ topological field theory $(2 \mathrm{~d}$ TFT) is obtained by twisting a $2 \mathrm{~d}$ supersymmetric field theory, then it is possible to regard topological Dbranes as a special class of "physical" D-branes (BPS D-branes). In fact, much of recent progress in string theory has resulted from studying BPS D-branes.

From the mathematical viewpoint, topological string theory is an alternative way of describing certain important geometric categories, such as the category of coherent sheaves on a Calabi-Yau manifold, and can serve as a powerful source of intuition. An outstanding example of such intuition is the Homological Mirror Symmetry conjecture [1].

Most works on topological string theory considered the case of topologically twisted $N=2$ sigma-models [2] with a Calabi-Yau target space. This is the case when the worldsheet theory is conformal, and topological correlators can also be interpreted in terms of a physical string theory [3]. However, one can also consider more general topologically twisted $N=2$ field theories and the corresponding D-branes. One class of such theories is given by sigma-models whose target is a Fano variety (say, a complex projective space, or a complex Grassmannian). Such QFTs, although conformally-invariant on the classical level, have non-trivial renormalization-group flow once quantum effects are taken into account. Another set of examples is provided by $N=2$ Landau-Ginzburg models (LG models) [ [ fact, in many cases these two classes of $N=2$ theories are related by mirror symmetry $[5$. For example, the sigma-model with target $\mathbb{C P}^{n}$ is mirror to a Landau-Ginzburg model with $n$ fields $x_{1}, \ldots, x_{n}$, taking values in $\mathbb{C}^{*}$, and a superpotential

$$
W=x_{1}+\cdots+x_{n}+\frac{1}{x_{1} \cdots x_{n}} .
$$

Thus if one wants to extend the Homological Mirror Symmetry conjecture to the nonCalabi-Yau case, one needs to understand D-branes in topologically twisted LG models. Note that all critical points of this superpotential are isolated and non-degenerate; this means that all the vacua have a mass gap, and the infrared limit of this LG model is trivial. In what follows we will call such LG models massive. Despite the triviality of the infrared limit, the Homological Mirror Symmetry conjecture remains meaningful and non-trivial in this case.

Very recently it has been proposed that massive $N=2 d=2$ QFTs can be used to describe certain non-standard superstring backgrounds with Ramond-Ramond flux [6]. Thus a study of D-branes in massive QFTs could be useful for understanding open strings in such Ramond-Ramond backgrounds.

In order to formulate our problem more concretely, let us first summarize the situation in the Calabi-Yau case, where the $N=2$ field theory is conformal. $N=2$ superconformal field theories have two topologically twisted versions: A-model and B-model [2, 3, 7]. The 
corresponding D-branes are called A-branes and B-branes. Mirror symmetry exchanges A-branes and B-branes. Tree-level topological correlators give the set of either A-branes or B-branes the structure of an $A_{\infty}$-category; gauge-invariant information is encoded by the corresponding derived categories. It has been argued that the derived category of B-branes is equivalent to the derived category of coherent sheaves [1, 8, 9]. A detailed check of this proposal has been performed in ref. [10].

For A-branes on Calabi-Yau manifolds, it has been proposed that the relevant $A_{\infty^{-}}$ category is the so-called Fukaya category, whose objects are (roughly) lagrangian submanifolds carrying vector bundles with flat connections [1]. Recently it has been shown that the derived Fukaya category is too small and does not accommodate certain physically acceptable A-branes [11. In particular, if we want the Homological Mirror Symmetry conjecture to be true for tori, then the Fukaya category must be enlarged with non-lagrangian (more specifically, coisotropic) branes.

In the case of Fano varieties, the sigma-model is not conformal. What is more important, the axial U(1) R-current is anomalous, and therefore one cannot define the B-twist [3. One can consider D-branes which preserve B-type supersymmetry, but the relation with the derived category of coherent sheaves is less straightforward [12, 13. Mirror symmetry relates B-branes on Fano varieties with A-branes in LG models. The latter have been studied from a variety of viewpoints in refs. [14, 15, 12, 16]. In the case when the Fano variety is $\mathbb{C P}^{n}$, the prediction of mirror symmetry has been tested in ref. [12, 17]. In particular, the mirrors of "exceptional" bundles on $\mathbb{C P}^{n}$ have been identified, and in the case of $\mathbb{C P}^{1}$ and $\mathbb{C P}^{2}$ it has been checked that morphisms between these bundles in the derived category of coherent sheaves on $\mathbb{C P}^{n}$ agree with the Floer homology between their mirror A-branes.

One can also consider the category of A-branes on a Fano manifold. Since the vector $\mathrm{U}(1) \mathrm{R}$-current is not anomalous, the A-twist is well-defined, and A-branes can be regarded as topological boundary conditions for the A-model. Presumably, the category of A-branes contains the derived Fukaya category as a subcategory, but other than that little is known about it, even in the case of $\mathbb{C P}^{n}$. If we assume mirror symmetry, we can learn about the category of A-branes on $\mathbb{C P}^{n}$ by studying B-branes in the mirror LG model. The B-twist is well-defined for any LG model whose target has a trivial canonical bundle, thus B-branes in such a LG model can be regarded as topological boundary conditions for the B-model. An obvious question is how the introduction of the superpotential deforms the relation between the category of B-branes and the derived category of coherent sheaves.

Important steps towards understanding B-branes in LG models have been taken in refs. [14, 12, 13, 16, 18] (see also refs. 19, 20, 21] for a related work). In these papers general properties of B-branes have been studied, and several concrete examples have been discussed. A somewhat surprising lesson from these works is that the category of B-branes remains non-trivial even in a massive LG model, where the bulk $2 \mathrm{~d}$ TFT is trivial. For example, if we take the superpotential $W$ to be a non-degenerate quadratic function on $\mathbb{C}^{n}$, the graded algebra of endomorphisms of a D0-brane sitting at the critical point of $W$ is isomorphic to a Clifford algebra with $n$ generators [16]. This raises the question if one can determine the category of B-branes in any massive LG model. 
A proposal which accomplishes this has been put forward by M. Kontsevich. Roughly speaking, the proposal is that the superpotential $W$ deforms the derived category of coherent sheaves by replacing complexes of locally free sheaves with "twisted" complexes. Here "twisted" means that compositions of successive morphisms in a complex are equal to $W$, instead of zero. One also needs to switch from $\mathbb{Z}$-graded complexes to $\mathbb{Z}_{2}$-graded ones. Kontsevich's proposal is supposed to describe B-branes in any LG model such that the critical set of $W$ is compact; in particular, it does not require the critical points of $W$ to be non-degenerate.

The main goal of this paper is to provide evidence for Kontsevich's proposal. Our evidence is of two kinds. First, we argue on physical grounds that twisted complexes arise as a consequence of BRST-invariance. More precisely, while in the presence of the superpotential a holomorphic vector bundle or a complex of vector bundles does not correspond to a B-type boundary condition, we show that any twisted complex of holomorphic vector bundles is a valid B-brane. Second, we test the proposal in some specific cases where morphisms between branes (i.e. spectra of topological open strings) can be easily computed. We focus on the massive case, where the proposal simplifies considerably. Namely, the category of $\mathbb{Z}_{2}$-graded twisted complexes can be related to the sum of several copies of the category of finite-dimensional $\mathbb{Z}_{2}$-graded modules over a Clifford algebra. We will denote the latter category $C_{\text {tot }}$ in what follows. This reformulation is helpful, because the functor from the category of B-branes in a massive LG model to $C_{\text {tot }}$ is very simple to describe. In this paper we perform some checks that this functor is an embedding of graded categories. In view of the above-mentioned "duality" between $\mathbb{Z}_{2}$-graded twisted complexes and $C_{\text {tot }}$, this provides a test of Kontsevich's proposal. Assuming the validity of the proposal, we infer that the category of B-branes in an arbitrary massive LG model is a full sub-category of $\mathrm{C}_{\text {tot }}$. The latter has a very simple and explicit description.

Since for many Fano varieties the mirror LG model is known, our results allow one to effectively compute the category of A-branes for such varieties. From the mathematical viewpoint, it is an interesting challenge to reproduce such results using methods of symplectic geometry.

Axiomatic definitions of topological D-branes for 2d Topological Field Theories $(2 \mathrm{~d}$ TFTs) have been recently proposed by G. Moore and G. Segal [22 and C. I. Lazaroiu [23]. One of the main unresolved problems in the axiomatic approach is whether these axioms determine unambiguously the category of topological D-branes associated to a given TFT. We show that the category of B-branes for a massive LG model with a quadratic superpotential provides a counter-example to uniqueness. In fact, this example shows that uniqueness, if understood naively, fails also for ordinary (i.e. non-topological) D-branes in any closed superstring background. However, this particular failure is rather mild, i.e. it does not seem to have serious physical consequences.

Now let us describe the content of the paper in more detail. In section 2 we recall some basic facts about mirror symmetry between Fano varieties and LG models. In section 1 we review general properties of B-branes in LG models. We argue that if the superpotential has only isolated critical points, then it is sufficient to study B-branes in the infinitesimal neighborhood of each critical point. For example, if all critical points of $W$ are non- 
degenerate, one does not lose anything if one replaces the superpotential by its quadratic approximation near each critical point. The material in this section is not new and has been previously discussed in refs. [12, 13, 16, 18]. In sections 1 and 5 we study B-branes in the LG model with the superpotential $W=x y$. This is the simplest LG model where B-branes of dimension larger than 0 are present. In section 5 we discuss B-branes in more general LG models with the superpotential $W=z_{1}^{2}+z_{2}^{2}+\cdots+z_{n}^{2}$. In section 7 we explain Kontsevich's proposal and show that our results are consistent with it. We also explain why BRST invariance of boundary conditions requires twisted complexes of vector bundles instead of ordinary complexes. This provides a physical explanation of Kontsevich's proposal. We also relate B-branes in massive LG models to $\mathbb{Z}_{2}$-graded Clifford modules. In section 8 we use Homological Mirror Symmetry to compute the category of A-branes for $\mathbb{C P}^{2}$ and $\mathbb{C P}^{1} \times \mathbb{C P}^{1}$. Section 9 contains concluding remarks.

\section{Mirror Symmetry for Fano varieties and LG models}

A Fano variety is a compact complex manifold whose anti-canonical line bundle is ample. This is equivalent to saying that the first Chern class of the canonical line bundle is negativedefinite. An $N=2$ sigma-model whose target space is a Fano variety describes an $N=2$ $d=2$ field theory which is free in the ultraviolet. In the infrared, it can either flow to a massive vacuum, or to a non-trivial $N=2 \mathrm{SCFT}$. Note that classically $N=2$ sigma-models have both vector and axial U(1) R-symmetries, but for Fano varieties quantum anomalies break the axial R-symmetry down to a discrete subgroup. Generically, this subgroup is $\mathbb{Z}_{2}$, but in special cases it can be larger. In the Calabi-Yau case the full axial R-symmetry is non-anomalous, and it is this fact that makes Calabi-Yau target spaces so special.

The simplest examples of Fano varieties are complex projective spaces $\mathbb{C P}^{n}$. The corresponding $N=2$ field theories are well studied; in fact, these models are integrable, in the sense that the exact S-matrix is known [24, 25]. These theories have only massive vacua. A more general set of examples is given by Grassmann varieties $G(n, k)$, which are defined as spaces of complex $k$-planes in an $n$-dimensional complex vector space. The corresponding $N=2$ field theories are also integrable [26].

An $N=2$ field theory which has a conserved vector (resp. axial) R-current admits a topological A-twist (resp. B-twist), which yields a 2d topological field theory called the A-model (resp. B-model). $N=2$ superconformal field theories have both axial and vector R-symmetries, and therefore admit both kinds of twisting. A-branes and B-branes are "defined" as boundary conditions which are consistent with A-twist and B-twist, respectively. ${ }^{1}$ These two sets of branes have the structure of a category. ${ }^{2}$ The space of morphisms is defined as the state space of topological open strings stretched between pairs of branes. Composition of morphisms is defined by means of 3-point correlators in topological open string theory. Since state spaces of open strings are graded vector spaces, brane categories

\footnotetext{
${ }^{1}$ We put the word "defined" in quotes because there is no generally accepted definition of a boundary condition for a $2 \mathrm{~d}$ field theory.

${ }^{2}$ The set of all D-branes is not a category in any natural sense. The reason is the presence of singular terms in the boundary operator product expansion.
} 
are graded categories. In the case of A-branes, spaces of morphisms are graded by the axial R-charge; in the case of B-branes, by the vector R-charge. For Fano varieties, the axial R-symmetry is generically $\mathbb{Z}_{2}$, so spaces of morphisms in the category of A-branes are $\mathbb{Z}_{2}$-graded vector spaces. In the Calabi-Yau case, the full axial U(1) R-symmetry is non-anomalous, and therefore the category of A-branes is $\mathbb{Z}$-graded. This is the reason one has to work with $\mathbb{Z}$-graded lagrangian submanifolds in the Calabi-Yau case [1]. In the Fano case, one only needs to require that lagrangian submanifolds be oriented.

In the Calabi-Yau case, we can also consider the category of B-branes. Since the vector R-current is non-anomalous, this category is $\mathbb{Z}$-graded. In the Fano case, the B-twist is not defined, and there is no obvious way to define the category of B-branes.

Given a graded category, one can enlarge it by adding for any object $Y$ its shifts $Y[i]$, where $i \in \mathbb{Z}$ or $i \in \mathbb{Z} / 2 \mathbb{Z}$, and defining morphisms as follows:

$$
\operatorname{Mor}^{k}\left(Y_{1}[i], Y_{2}[j]\right)=\operatorname{Mor}^{k+j-i}\left(Y_{1}, Y_{2}\right) .
$$

In string theory, the R-charge of strings connecting two different branes is defined only up to an integer constant; changing this constant by $k$ shifts the degree of all morphisms by $k$. The effect of this arbitrariness is that for any brane $Y$ its shifts $Y[i]$ are automatically included. This implies that no information is lost if we replace groups of morphisms with their degree-0 components. This is what one usually does when working with categories of complexes, such as the derived category. Nevertheless, in this paper we will keep morphisms of all degrees, since this conforms better to physical conventions. From this viewpoint, the mathematical counterpart of the category of B-branes on a Calabi-Yau $X$ is not $D^{b}(\operatorname{Coh}(X))$, but a $\mathbb{Z}$-graded category which is called the completion of $D^{b}(\operatorname{Coh}(X))$ with respect to the shift functor.

For a sigma-model on a Calabi-Yau manifold which is a complete intersection in a toric variety, the mirror theory is again a sigma-model of the same kind. For Fano varieties which are complete intersections in a toric variety, the mirror theory is a LG model whose target is a non-compact Calabi-Yau [5]. A general definition of a LG model involves, besides a choice of a target manifold, a choice of a holomorphic function $W$ on this manifold (the superpotential). Thus non-trivial LG models require non-compact target spaces. This non-compactness usually does not cause trouble: the important thing is for the critical set of $W$ to be compact. In general, superpotential breaks vector R-symmetry down to $\mathbb{Z}_{2}$. Thus the A-twist is not defined, in general. On the other hand, since the canonical bundle of the target manifold is trivial, the axial R-symmetry is not anomalous, and the B-twist is well-defined. We expect that the category of A-branes on a Fano variety is equivalent to the category of B-branes on the mirror LG model.

For example, the mirror of $\mathbb{C P} \mathbb{P}^{n}$ is a LG model whose target is $\left(\mathbb{C}^{*}\right)^{n}$ with the superpotential eq. (1.1). This superpotential has $n+1$ non-degenerate critical points given by

$$
x_{1}=x_{2}=\cdots=x_{n}=e^{2 \pi i k /(n+1)}, \quad k=0, \ldots, n .
$$

The physical interpretation is that the theory has $n+1$ massive vacua. This agrees with the count of vacua in the $\mathbb{C P}^{n}$ model. Furthermore, the superpotential breaks vector $\mathrm{U}(1)_{R}$ 
symmetry down to $\mathbb{Z}_{2}$ given by

$$
\theta_{+} \rightarrow-\theta_{+}, \quad \theta_{-} \rightarrow-\theta_{-},
$$

where $\theta_{ \pm}$are the usual odd coordinates on the chiral $(2,2)$ superspace. As a consequence, spaces of morphisms in the category of B-branes in this LG model are $\mathbb{Z}_{2}$-graded. This is mirror to the fact that morphisms in the category of A-branes on $\mathbb{C P}^{n}$ are $\mathbb{Z}_{2}$-graded. ${ }^{3}$

As a rule, it is easier to understand B-branes, rather than A-branes. Therefore, we now turn to the study of B-branes in LG models, in the hope that it will illuminate the properties of A-branes on Fano varieties.

\section{General properties of B-branes in LG models}

The classical geometry of B-branes was described in refs. [14, 12] (see also ref. [27]). In this section we summarize the results of refs. [14, 12] which are relevant for us and discuss some simple consequences.

Let $X$ be the target space of a LG model. On general grounds, it must be Kähler manifold (possibly non-compact). Let the $W$ be a fixed holomorphic function on $X$ (the superpotential). Let $Y$ be a submanifold of $X$, and let $E$ be a hermitean vector bundle over $Y$ with a unitary connection $\nabla$. The rank of $E$ will be called the multiplicity of the corresponding D-brane. It is shown in ref. [12] that the triple $(Y, E, \nabla)$ defines a classical B-type boundary condition if and only if $Y$ is a complex submanifold of $X, W$ is constant on $Y$, and the pair $(E, \bar{\partial})$, where $\bar{\partial}$ is the anti-holomorphic part of $\nabla$, is a holomorphic vector bundle. For example, a point on $X$ together with a choice of multiplicity $r \in \mathbb{N}$ defines a B-type boundary condition.

The class of B-branes described in the previous paragraph does not exhaust all possible B-branes. But it appears plausible that all B-branes can be obtained as bound states of the branes described above.

It was noticed in ref. [16] (see also ref. [18]) that most of the "classical" B-branes should be regarded as zero objects in the category of B-branes. A classical B-brane is isomorphic to the zero object if and only if the space of its endomorphisms is zero-dimensional, i.e. when there are no supersymmetric open string states connecting the brane with itself. In this case one says that world-sheet supersymmetry is spontaneously broken. For example, it is explained in refs. [13, 16, 18 that if $Y$ is a point on $X$ which is not a critical point of $W$, then B-type supersymmetry is spontaneously broken, and therefore $Y$ is isomorphic to the zero object in the category of B-branes.

This phenomenon reduces enormously the number of B-branes that one needs to consider, and makes it plausible that the whole category can be described combinatorially, using only the number and type of critical points of $W$. To substantiate this claim, we first notice that to any B-brane in the class described above one can assign a complex number, the value of $W$ on this brane. Further, there are no non-zero morphisms between branes

\footnotetext{
${ }^{3}$ In this LG model, there is in fact an unbroken $\mathbb{Z}_{2(n+1)}$ R-symmetry; the $\mathbb{Z}_{2}$ symmetry discussed in the text is its subgroup. This is mirror to the fact that the $\mathbb{C P}^{n}$ sigma-model has non-anomalous axial $\mathbb{Z}_{2(n+1)}$ $\mathrm{R}$-symmetry. In this paper we will only keep track of $\mathbb{Z}_{2}$-gradings.
} 
with different values of $W$, because any string connecting such branes will have non-zero energy and will not be supersymmetric [13, 18]. (Unlike in the case of A-branes, there is no central charge in the supersymmetry algebra, and supersymmetric states must have zero energy.) Thus the category of B-branes can be regarded as a family of categories parametrized by $\mathbb{C}$, and the categories at different points in $\mathbb{C}$ do not "talk" to each other.

Second, zero-energy classical configurations of an open string must be constant maps from the interval to $Y$, such that the potential energy $|\partial W|^{2}$ vanishes. This implies that unless a B-brane passes through a critical point of $W$, there are no supersymmetric states for strings connecting this B-brane to any other B-brane (including itself). It follows that categories corresponding to non-critical values of $W$ are trivial (contain only the zero object).

Now let us assume that all critical points of $W$ are isolated. By scaling up the Kähler form, we can make the semi-classical approximation arbitrarily good. This means that wave-functions of all string states will be arbitrarily well localized near a particular critical point of $W$, and the overlap between wave-functions associated to different critical points will be arbitrarily small. Since topological correlators do not depend on the Kähler form, it is clear that morphisms between B-branes can be computed using only the leading terms in the Taylor expansion of $W$ around the critical points. ${ }^{4}$

To be more precise, we can attach a category to each isolated critical point of $W$ as follows: we replace $W$ by a polynomial which has the same singularity, and consider the category of B-branes on an affine space with this polynomial superpotential. Now let us form the direct sum of such categories over all critical points of $W$ and call it $C_{\text {tot }}$. There is an obvious map which associates to any B-brane an object of $C_{\text {tot }}$. Invariance of topological correlators under variations of the Kähler form means that this map extends to a functor, and this functor is full and faithful. In other words, the category of B-branes is a full sub-category of $C_{\text {tot }}$.

In particular, when all critical points of $W$ are non-degenerate (i.e. when all vacua are massive), the problem reduces to understanding B-branes in the LG model with target $\mathbb{C}^{n}$ and superpotential

$$
W=z_{1}^{2}+\cdots+z_{n}^{2} .
$$

The corresponding bulk theory is free, but since the boundary conditions need not be linear, the problem of determining all B-branes is far from trivial. In this paper we will study B-branes which correspond to linear boundary conditions. Some such branes have been considered in refs. [16, 13, 18]. We will see below that if Kontsevich's conjecture is true, then these branes generate the whole category of B-branes.

Note that the LG superpotential eq. (1.1) satisfies the conditions stated above. Thus, assuming mirror symmetry, we can gain information about A-branes on $\mathbb{C P}^{n}$ by studying B-branes in the free LG model with the superpotential eq. (3.1). In the case $n=1$ this has been done in ref. [16]; in that case the category of A-branes is independently known, and one can see that the mirror conjecture holds true. In section 8 we discuss the less trivial case $n=2$.

\footnotetext{
${ }^{4}$ In fact, in refs. [5, 16, 18] there is a proposal how to compute spaces of morphisms between B-branes using a deformation of the Dolbeault complex by $\partial W$.
} 
Before continuing, let us make some further comments on the relation between $\mathrm{C}_{\text {tot }}$ and the category of B-branes. We do not claim that the two categories are equivalent, only that the latter is a full sub-category of the former. This means that each B-brane can be regarded as a direct sum of "local" B-branes attached to critical points, but not every direct sum is a valid B-brane. We will see in section 8 some examples where the category of B-branes is strictly smaller than $C_{\text {tot }}$. Nevertheless, since each B-brane behaves as a composite of "local" B-branes, it is reasonable to enlarge the category of B-branes by allowing arbitrary sums of "local" B-branes. Then the category of B-branes becomes equivalent to $\mathrm{C}_{\text {tot }}$. From a purely algebraic standpoint, this is a very natural procedure (c.f. a discussion in ref. [23] concerning reducible and irreducible branes), but the drawback is that the new branes lack a clear geometric interpretation. section 8 contains a further discussion of this issue.

\section{B-Branes in the LG model with $W=x y$}

\subsection{Preliminaries}

We begin by recalling the results of ref. [16] concerning B-branes in the simplest LG model with the superpotential $W=z^{2}$. In this case, the only allowed B-branes are D0-branes located at $z=0$. It has been shown in ref. [16] that the space of endomorphisms of a single D0-brane is two-dimensional, with one-dimensional even subspace and one-dimensional odd subspace. As a graded algebra, it is generated over $\mathbb{C}$ by the identity and an odd element $\theta$ with the relation

$$
\theta^{2}=1
$$

This is a Clifford algebra $\mathrm{Cl}(1, \mathbb{C})$. If we take $N$ D0-branes, then the algebra of endomorphisms becomes

$$
\mathrm{Cl}(1, \mathbb{C}) \otimes \operatorname{Mat}(N, \mathbb{C}),
$$

where $\operatorname{Mat}(N, \mathbb{C})$ is the algebra of $N \times N$ complex matrices.

We are interested in the next simplest LG model with the superpotential $W=x y$. Again, D0-branes must be localized at $x=y=0$, and it has been shown in ref. [16] that the algebra of endomorphisms of a single D0-brane is generated by two odd elements $\theta_{1}, \theta_{2}$ with the relations

$$
\theta_{1} \theta_{2}+\theta_{2} \theta_{1}=1, \quad\left(\theta_{1}\right)^{2}=\left(\theta_{2}\right)^{2}=0 .
$$

This is a Clifford algebra $\mathrm{Cl}(2, \mathbb{C})$. More invariantly, if we denote by $V$ the complex vector space which is the target space of our LG model, we can say that fermion zero modes for the open string take values in $V$. The hessian of the superpotential

$$
Q_{i j}=\partial_{i} \partial_{j} W(0)
$$

defines a non-degenerate symmetric bilinear form on $V$, and the endomorphism algebra of the D0-brane is the Clifford algebra associated to the pair $(V, Q)$. (Some standard facts about Clifford algebras and their modules are described in the eppendix. We will freely use these facts in what follows.) 
But in this case there can also be B-branes of higher dimension, namely D2-branes. (This is briefly discussed in ref. [18].) Irreducible D2-branes are irreducible components of the critical level set $W=0$, which is a singular quadric

$$
x y=0 .
$$

Thus there are two candidate irreducible D2-branes, given by $x=0$ and $y=0$, respectively. Our immediate goal is to compute their endomorphisms, as well as morphisms between D2branes and D0-branes. In physical terms, we will compute the spectrum and disk correlators of the topological open string with appropriate boundary conditions.

\subsection{Equations of motion and SUSY transformations}

We consider the Landau-Ginzburg model on $\mathbb{R} \times[0, \pi]$ with two chiral superfields $\Phi_{1}$ and $\Phi_{2}$. The superpotential assumes the following form

$$
W(\Phi)=2 m \Phi_{1} \Phi_{2}
$$

We include a positive factor $2 m$ in the superpotential in order to keep track of dimensions of topological correlators later. In physical terms, $m$ is a measure of the mass gap in the Landau-Ginzburg model.

Assuming the standard Kähler potential $K=\left|\Phi_{1}\right|^{2}+\left|\Phi_{2}\right|^{2}$, the world-sheet action reads

$$
\begin{aligned}
S=\frac{1}{2 \pi} \int_{\mathbb{R} \times[0, \pi]} \mathrm{d}^{2} x & \left\{\sum_{\alpha=1}^{2}\left(\left|\partial_{t} \phi^{\alpha}\right|^{2}-\left|\partial_{\sigma} \phi^{\alpha}\right|^{2}+i \psi_{-}^{\bar{\alpha}} \partial_{+} \psi_{-}^{\alpha}+i \psi_{+}^{\bar{\alpha}} \partial_{-} \psi_{+}^{\alpha}\right)-\left|m \phi^{1}\right|^{2}-\right. \\
& \left.-\left|m \phi^{2}\right|^{2}-m\left(\psi_{+}^{1} \psi_{-}^{2}+\psi_{+}^{2} \psi_{-}^{1}\right)-\bar{m}\left(\psi_{-}^{\overline{1}} \psi_{+}^{\overline{2}}+\psi_{-}^{\overline{2}} \psi_{+}^{\overline{1}}\right)\right\}
\end{aligned}
$$

where $\phi^{\alpha}$ are the bosonic components of $\Phi_{\alpha}$, and $\psi^{\alpha}$ are their fermionic partners. The world-sheet parametrization $(t, \sigma)$ is such that $t$ is the world-sheet time.

From the bosonic lagrangian density

$$
\mathcal{L}_{B}=\sum_{\alpha=1,2}\left(\left|\dot{\phi}_{\alpha}\right|^{2}-\left|\phi_{\alpha}^{\prime}\right|^{2}-m^{2}\left|\phi_{\alpha}\right|^{2}\right)
$$

one readily obtains the EOM's for $\phi$

$$
\ddot{\phi}_{\alpha}-\phi_{\alpha}^{\prime \prime}+m^{2} \phi_{\alpha}=0, \quad \alpha=1,2 .
$$

Similarly, in terms of new variables

$$
b_{\alpha}=\frac{\psi_{-}^{\alpha}+\psi_{+}^{\alpha}}{\sqrt{2}}, \quad c_{\alpha}=\frac{\psi_{-}^{\alpha}-\psi_{+}^{\alpha}}{\sqrt{2}}, \quad \alpha=1,2
$$


the fermionic lagrangian density can be written as

$$
\begin{aligned}
\mathcal{L}_{F}= & i \bar{b}_{1} \dot{b}_{1}+i \bar{b}_{2} \dot{b}_{2}+i \bar{c}_{1} \dot{c}_{1}+i \bar{c}_{2} \dot{c}_{2}+ \\
& +\bar{b}_{1}\left(i \partial_{\sigma} c_{1}+\bar{m} \overline{c_{2}}\right)+\left(-i \partial_{\sigma} \bar{c}_{1}+m c_{2}\right) b_{1}+ \\
& +\bar{b}_{2}\left(i \partial_{\sigma} c_{2}+\bar{m} \overline{c_{1}}\right)+\left(-i \partial_{\sigma} \bar{c}_{2}+m c_{1}\right) b_{2} .
\end{aligned}
$$

The fermionic EOM's are given by

$$
\begin{aligned}
& i \dot{b}_{1}+i c_{1}^{\prime}+m \bar{c}_{2}=0, \\
& i \dot{b}_{2}+i c_{2}^{\prime}+m \bar{c}_{1}=0, \\
& i \dot{c}_{1}+i b_{1}^{\prime}-m \bar{b}_{2}=0, \\
& i \dot{c}_{2}+i b_{2}^{\prime}-m \bar{b}_{1}=0 .
\end{aligned}
$$

B-type supersymmetry transformations are well known (see, for example, ref. [12]) and look as follows:

$$
\begin{array}{lll}
\delta \phi_{1}=\sqrt{2} \epsilon b 1_{1}, & \delta b_{1}=-\sqrt{2} i \bar{\epsilon} \dot{\phi}_{1}, & \delta c_{1}=\sqrt{2} i \bar{\epsilon} \phi_{1}^{\prime}+\sqrt{2} \epsilon \bar{m} \bar{\phi}_{2}, \\
\delta \phi_{2}=\sqrt{2} \epsilon b_{2}, & \delta b_{2}=-\sqrt{2} i \bar{\epsilon} \dot{\phi}_{2}, & \delta c_{2}=\sqrt{2} i \bar{\epsilon} \phi_{2}^{\prime}+\sqrt{2} \epsilon \bar{m} \bar{\phi}_{1} .
\end{array}
$$

Now we will perform canonical quantization of this system with various boundary conditions which correspond to D0-D0 strings, D2-D2 strings, and D0-D2 strings.

\subsection{Spectrum of D0-D0 strings}

We would like to find supersymmetric states of D0-D0 strings, since these correspond to endomorphisms of the D0-brane. The relevant boundary conditions are

$$
\left\{\begin{array}{l}
\phi_{1}=\phi_{2}=0 \\
b_{1}=b_{2}=0
\end{array} \quad \sigma=0, \pi .\right.
$$

First consider the bosonic degrees of freedom. The boundary conditions give the following mode expansions for $\phi$ and its conjugate momentum $\pi_{\phi}$ :

$$
\begin{aligned}
\phi_{\alpha} & =\frac{1}{\sqrt{\pi}} \sum_{n=1}^{\infty} \frac{i}{\sqrt{\omega_{n}}}\left(a_{\alpha, n}-\tilde{a}_{\alpha, n}^{\dagger}\right) \sin n \sigma \\
\pi_{\phi_{\alpha}} & =\partial_{t} \bar{\phi}=\frac{1}{\sqrt{\pi}} \sum_{n=1}^{\infty} \sqrt{\omega_{n}}\left(\tilde{a}_{\alpha, n}+a_{\alpha, n}^{\dagger}\right) \sin n \sigma
\end{aligned}
$$

where

$$
\omega_{n}=\sqrt{n^{2}+m^{2}}
$$


To quantize, we impose the following commutation relations:

$$
\left[a_{\alpha, n}, a_{\beta, m}^{\dagger}\right]=\left[\tilde{a}_{\alpha, n}, \tilde{a}_{\beta, m}^{\dagger}\right]=\delta_{\alpha \beta} \delta_{m n},
$$

with all other commutators vanishing. It is easy to check that (4.5) is compatible with the following canonical commutation relations

$$
\left[\phi_{\alpha}(\sigma), \pi_{\phi_{\beta}}\left(\sigma^{\prime}\right)\right]=i \delta_{\alpha \beta} \cdot \frac{2}{\pi} \sum_{n=1}^{\infty} \sin n \sigma \sin n \sigma^{\prime}=i \delta_{\alpha \beta} \delta\left(\sigma-\sigma^{\prime}\right)
$$

The bosonic hamiltonian is given by

$$
\begin{aligned}
H_{B} & =\int \mathrm{d} \sigma \sum_{\alpha=1,2}\left(\left|\dot{\phi}_{\alpha}\right|^{2}+\left|\phi_{\alpha}^{\prime}\right|^{2}+m^{2}\left|\phi_{\alpha}\right|^{2}\right) \\
& =\sum_{n=1}^{\infty} \omega_{n}\left(a_{1, n}^{\dagger} a_{1, n}+\tilde{a}_{1, n}^{\dagger} \tilde{a}_{1, n}+a_{2, n}^{\dagger} a_{2, n}+\tilde{a}_{2, n}^{\dagger} \tilde{a}_{2, n}+2\right)
\end{aligned}
$$

where the additive constant " +2 " in the sum is the bosonic zero point energy. We shall see later that it is exactly canceled by the fermionic zero point energy.

Next we consider the fermionic degrees of freedom. It will turn out convenient to use the following combinations as new dynamical variables:

$$
b_{ \pm}=\frac{b_{1} \pm i \bar{b}_{2}}{\sqrt{2}}, \quad c_{ \pm}=\frac{c_{1} \pm i \bar{c}_{2}}{\sqrt{2}} .
$$

The main advantage of using $b_{ \pm}$and $c_{ \pm}$is that the EOM's for the unbarred quantities are decoupled from those for the barred quantities. The mode expansions for these fields have the following form:

$$
\begin{aligned}
& b_{+}=\frac{i}{\sqrt{\pi}} \sum_{n=1}^{\infty} \frac{n+i m}{\omega}\left(\alpha_{2, n}-\tilde{\alpha}_{2, n}^{\dagger}\right) \sin n \sigma \\
& b_{-}=\frac{i}{\sqrt{\pi}} \sum_{n=1}^{\infty} \frac{n-i m}{\omega}\left(\alpha_{1, n}-\tilde{\alpha}_{1, n}^{\dagger}\right) \sin n \sigma \\
& c_{+}=\frac{1}{\sqrt{\pi}} \sum_{n=1}^{\infty} \frac{1}{n-i m}(n \cos n \sigma+m \sin n \sigma)\left(\alpha_{2, n}+\tilde{\alpha}_{2, n}^{\dagger}\right)+\lambda_{+} e^{m \sigma}, \\
& c_{-}=\frac{1}{\sqrt{\pi}} \sum_{n=1}^{\infty} \frac{1}{n+i m}(n \cos n \sigma-m \sin n \sigma)\left(\alpha_{1, n}+\tilde{\alpha}_{1, n}^{\dagger}\right)+\lambda_{-} e^{-m \sigma} .
\end{aligned}
$$

To fix the commutation rules for the oscillators we impose the canonical commutation relations for $b$ :

$$
\begin{aligned}
\left\{b_{+}, b_{+}\right\}=\left\{b_{-}, b_{-}\right\}=\left\{b_{+}, b_{-}\right\}=\left\{b_{+}, \bar{b}_{-}\right\}=\left\{b_{-}, \bar{b}_{+}\right\} & =0 \\
\left\{b_{+}(\sigma), \bar{b}_{+}\left(\sigma^{\prime}\right)\right\}=\left\{b_{-}(\sigma), \bar{b}_{-}\left(\sigma^{\prime}\right)\right\} & =\delta\left(\sigma-\sigma^{\prime}\right) .
\end{aligned}
$$

A convenient choice of compatible commutation rules for oscillators is

$$
\left\{\alpha_{i, n}, \alpha_{i^{\prime}, n^{\prime}}^{\dagger}\right\}=\left\{\tilde{\alpha}_{i, n}, \tilde{\alpha}_{i^{\prime}, n^{\prime}}^{\dagger}\right\}=\delta_{i i^{\prime}} \delta_{n n^{\prime}}
$$


with all others vanishing. One can easily check that the canonical commutation relations for $c$ fields are also respected, provided that the following relations are imposed:

$$
\begin{aligned}
\{\zeta, \zeta\}=\{\eta, \eta\}=\{\zeta, \eta\} & =\left\{\zeta, \eta^{\dagger}\right\}=0 \\
\left\{\zeta, \zeta^{\dagger}\right\} & =\left\{\eta, \eta^{\dagger}\right\}=1
\end{aligned}
$$

where $\eta$ and $\zeta$ are defined by

$$
\lambda_{+} \equiv \zeta \sqrt{\frac{m}{\sinh m \pi}} e^{-\pi m / 2}, \quad \lambda_{-} \equiv \eta \sqrt{\frac{m}{\sinh m \pi}} e^{\pi m / 2} .
$$

The fermionic hamiltonian is

$$
\begin{aligned}
H_{F} & =\int_{0}^{\pi} \mathrm{d} \sigma\left[\left(i c_{1}^{\prime}+m \bar{c}_{2}\right) \bar{b}_{1}+\left(i c_{2}^{\prime}+m \bar{c}_{1}\right) \bar{b}_{2}+\text { h.c. }\right] \\
& =\int_{0}^{\pi} \mathrm{d} \sigma\left[i\left(c_{+}^{\prime}-m c_{+}\right) \bar{b}_{+}+i\left(c_{-}^{\prime}+m c_{-}\right) \bar{b}_{-}+\text {h.c. }\right] \\
& =\sum_{n=1}^{\infty} \omega_{n}\left(\alpha_{1, n}^{\dagger} \alpha_{1, n}+\alpha_{2, n}^{\dagger} \alpha_{2, n}+\tilde{\alpha}_{1, n}^{\dagger} \tilde{\alpha}_{1, n}+\tilde{\alpha}_{2, n}^{\dagger} \tilde{\alpha}_{2, n}-2\right) .
\end{aligned}
$$

Note that the additive constant -2 cancels the bosonic zero point energy. The hamiltonian is diagonalized in the Fock basis, and the zero-energy states are

$$
|0\rangle, \quad \zeta^{\dagger}|0\rangle, \quad \eta^{\dagger}|0\rangle, \quad \eta^{\dagger} \zeta^{\dagger}|0\rangle .
$$

The supercharge $Q$ can also be expanded in terms of oscillators. It can be shown that each term in the expansion contains an annihilation operator for non-zero modes, and that $Q$ does not depend on zero-mode oscillators $\zeta$ and $\eta$. Therefore $Q$ annihilates all four ground states.

\subsection{Spectrum of D2-D2 strings}

Since we have two different D2-branes related by a symmetry, there are two inequivalent possibilities: either our string begins and ends on the same D2-brane, or it begins on one D2brane, and ends on the other D2-brane. The first situation corresponds to endomorphisms of a D2-brane, while the second one corresponds to morphisms from one D2-brane to the other one.

First we consider the case when both boundaries end on the same brane, say, the one given by the equation $\Phi_{1}=0$. The relevant boundary conditions are

$$
\left\{\begin{array} { l } 
{ \phi _ { 1 } = 0 } \\
{ b _ { 1 } = 0 }
\end{array} \quad \text { and } \quad \left\{\begin{array}{l}
\partial_{\sigma} \phi_{2}=0 \\
c_{2}=0
\end{array} \quad \text { at } \sigma=0, \pi .\right.\right.
$$

First let us look at the bosons. The mode expansions for $\phi_{1}$ and its conjugate momentum are the same as before, while for $\phi_{2}$ and its conjugate momentum they are given by

$$
\phi_{2}=\frac{1}{\sqrt{2 \pi m}}\left(a_{2,0}+\tilde{a}_{2,0}^{\dagger}\right)+\sum_{n=1}^{\infty} \frac{1}{\sqrt{\pi \omega_{n}}}\left(a_{2, n}+\tilde{a}_{2, n}^{\dagger}\right) \cos n \sigma,
$$




$$
\pi_{\phi_{2}}=i \sqrt{\frac{m}{2 \pi}}\left(a_{2,0}^{\dagger}-\tilde{a}_{2,0}\right)+i \sum_{n=1}^{\infty} \sqrt{\frac{\omega_{n}}{\pi}}\left(a_{2, n}^{\dagger}-\tilde{a}_{2, n}\right) \cos n \sigma .
$$

Canonical commutation relations for bosonic fields imply the commutation relations eq. (4.5) for the oscillators. In terms of oscillators the bosonic hamiltonian is

$$
H_{B}=\sum_{n=1}^{\infty} \omega_{n}\left(a_{1, n}^{\dagger} a_{1, n}+\tilde{a}_{1, n}^{\dagger} \tilde{a}_{1, n}+1\right)+\sum_{n=0}^{\infty} \omega_{n}\left(a_{2, n}^{\dagger} a_{2, n}+\tilde{a}_{2, n}^{\dagger} \tilde{a}_{2, n}+1\right) .
$$

For the fermions, the mode expansions are given by

$$
\begin{aligned}
b_{1}= & \frac{i}{\sqrt{2 \pi}} \sum_{n=1}^{\infty}\left[\frac{n+i m}{\omega_{n}}\left(\alpha_{2, n}-\tilde{\alpha}_{2, n}^{\dagger}\right)+\frac{n-i m}{\omega_{n}}\left(\alpha_{1, n}-\tilde{\alpha}_{1, n}^{\dagger}\right)\right] \sin n \sigma, \\
b_{2}= & \frac{-1}{\sqrt{2 \pi}}\left(\tilde{\alpha}_{0}-\alpha_{0}^{\dagger}\right)+ \\
& +\frac{-i}{\sqrt{2 \pi}} \sum_{n=1}^{\infty}\left[-\frac{n+i m}{\omega_{n}}\left(\tilde{\alpha}_{1, n}-\alpha_{1, n}^{\dagger}\right)+\frac{n-i m}{\omega_{n}}\left(\tilde{\alpha}_{2, n}-\alpha_{2, n}^{\dagger}\right)\right] \cos n \sigma, \\
c_{1}= & \frac{1}{\sqrt{2 \pi}}\left(\alpha_{0}+\tilde{\alpha}_{0}^{\dagger}\right)+\frac{1}{\sqrt{2 \pi}} \sum_{n=1}^{\infty}\left(\alpha_{1, n}+\alpha_{2, n}+\tilde{\alpha}_{1, n}^{\dagger}+\tilde{\alpha}_{2, n}^{\dagger}\right) \cos n \sigma, \\
c_{2}= & \frac{1}{\sqrt{2 \pi}} \sum_{n=1}^{\infty}\left(\tilde{\alpha}_{2, n}-\tilde{\alpha}_{1, n}+\alpha_{2, n}^{\dagger}-\alpha_{1, n}^{\dagger}\right) \sin n \sigma .
\end{aligned}
$$

The canonical commutation relations for the fields $b_{i}$ and $c_{i}$ are equivalent to the following commutation relations for the oscillators:

$$
\begin{aligned}
\left\{\alpha_{i, n}, \alpha_{i^{\prime}, n^{\prime}}^{\dagger}\right\} & =\left\{\tilde{\alpha}_{i, n}, \tilde{\alpha}_{i^{\prime}, n^{\prime}}^{\dagger}\right\}=\delta_{i i^{\prime}} \delta_{n n^{\prime}}, \quad n=1,2, \ldots, \\
\left\{\alpha_{0}, \alpha_{0}^{\dagger}\right\} & =\left\{\tilde{\alpha}_{0}, \tilde{\alpha}_{0}^{\dagger}\right\}=1
\end{aligned}
$$

with all others vanishing. The fermionic hamiltonian can be shown to be

$$
\begin{aligned}
H_{F}= & \int_{0}^{\pi} \mathrm{d} \sigma\left[\left(i c_{1}^{\prime}+m \bar{c}_{2}\right) \bar{b}_{1}+\left(i c_{2}^{\prime}+m \bar{c}_{1}\right) \bar{b}_{2}+\text { h.c. }\right] \\
= & \sum_{n=1}^{\infty} \omega_{n}\left(\alpha_{1, n}^{\dagger} \alpha_{1, n}+\alpha_{2, n}^{\dagger} \alpha_{2, n}+\tilde{\alpha}_{1, n}^{\dagger} \tilde{\alpha}_{1, n}+\tilde{\alpha}_{2, n}^{\dagger} \tilde{\alpha}_{2, n}-2\right)+ \\
& +m\left(\alpha_{0}^{\dagger} \alpha_{0}+\tilde{\alpha}_{0}^{\dagger} \tilde{\alpha}_{0}-1\right) .
\end{aligned}
$$

The fermionic zero point energy cancels the bosonic zero point energy, and we see that there is a unique state with zero energy: the Fock vacuum. For the same reason as in the D0-D0 case, this state is supersymmetric (is annihilated by the supercharge).

Now consider the case when one end of the string $(\sigma=0)$ is attached to $\Phi_{1}=0$, and the other one $(\sigma=\pi)$ is attached to $\Phi_{2}=0$. The boundary conditions are

$$
\left\{\begin{array} { l } 
{ \phi _ { 1 } ( 0 ) = \partial _ { \sigma } \phi _ { 1 } ( \pi ) = 0 } \\
{ b _ { 1 } ( 0 ) = c _ { 1 } ( \pi ) = 0 }
\end{array} \quad \text { and } \quad \left\{\begin{array}{l}
\partial_{\sigma} \phi_{2}(0)=\phi_{2}(\pi)=0 \\
c_{2}(0)=b_{2}(\pi)=0
\end{array} .\right.\right.
$$


The mode expansions for the bosons are

$$
\begin{aligned}
\phi_{1} & =\sum_{n=1}^{\infty} \frac{i}{\sqrt{\pi \omega_{n}}}\left(a_{1, n}-\tilde{a}_{1, n}^{\dagger}\right) \sin k_{n} \sigma, \\
\pi_{\phi_{1}} & =\sum_{n=1}^{\infty} \sqrt{\frac{\omega_{n}}{\pi}}\left(\tilde{a}_{1, n}+a_{1, n}^{\dagger}\right) \sin k_{n} \sigma, \\
\phi_{2} & =\sum_{n=1}^{\infty} \frac{1}{\sqrt{\pi \omega_{n}}}\left(a_{2, n}+\tilde{a}_{2, n}^{\dagger}\right) \cos k_{n} \sigma, \\
\pi_{\phi_{2}} & =\sum_{n=1}^{\infty} i \sqrt{\frac{\omega_{n}}{\pi}}\left(-\tilde{a}_{1, n}+a_{1, n}^{\dagger}\right) \cos k_{n} \sigma,
\end{aligned}
$$

while for the fermions they are

$$
\begin{aligned}
& b_{1}=\frac{i}{\sqrt{2 \pi}} \sum_{n=1}^{\infty}\left[\frac{k_{n}+i m}{\omega_{n}}\left(\alpha_{2, n}-\tilde{\alpha}_{2, n}^{\dagger}\right)+\frac{k_{n}-i m}{\omega_{n}}\left(\alpha_{1, n}-\tilde{\alpha}_{1, n}^{\dagger}\right)\right] \sin k_{n} \sigma \\
& b_{2}=\frac{1}{\sqrt{2 \pi}} \sum_{n=1}^{\infty}\left[\frac{k_{n}+i m}{\omega_{n}}\left(\tilde{\alpha}_{1, n}-\alpha_{1, n}^{\dagger}\right)-\frac{k_{n}-i m}{\omega_{n}}\left(\tilde{\alpha}_{2, n}-\alpha_{2, n}^{\dagger}\right)\right] \cos k_{n} \sigma \\
& c_{1}=\frac{1}{\sqrt{2 \pi}} \sum_{n=1}^{\infty}\left(\alpha_{1, n}+\alpha_{2, n}+\tilde{\alpha}_{1, n}^{\dagger}+\tilde{\alpha}_{2, n}^{\dagger}\right) \cos k_{n} \sigma \\
& c_{2}=\frac{i}{\sqrt{2 \pi}} \sum_{n=1}^{\infty}\left(\tilde{\alpha}_{1, n}-\tilde{\alpha}_{2, n}+\alpha_{1, n}^{\dagger}-\alpha_{2, n}^{\dagger}\right) \sin k_{n} \sigma
\end{aligned}
$$

where

$$
k_{n}=n-\frac{1}{2}, \quad \omega_{n}=\sqrt{k_{n}^{2}+m^{2}} .
$$

One can show as before that commutation relations (4.5) and (4.8) yield all the canonical commutation relations, and the total hamiltonian is diagonalized in the Fock basis as follows:

$$
H=\sum_{i=1}^{2} \sum_{n=0}^{\infty} \omega_{n}\left(a_{i, n}^{\dagger} a_{i, n}+\tilde{a}_{i, n}^{\dagger} \tilde{a}_{i, n}+\alpha_{i, n}^{\dagger} \alpha_{i, n}+\tilde{\alpha}_{i, n}^{\dagger} \tilde{\alpha}_{i, n}\right) .
$$

Again there is a single ground state which is annihilated by the supercharge.

\subsection{Spectrum of D0-D2 strings}

The boundary conditions for the bosons are

$$
\phi_{1}(0)=\phi_{1}(\pi)=0, \quad \phi_{2}(0)=\partial_{\sigma} \phi_{2}(\pi)=0 .
$$

The corresponding mode expansions are

$$
\begin{aligned}
\phi_{1} & =\sum_{n=1}^{\infty} \frac{i}{\sqrt{\pi \omega_{1, n}}}\left(a_{1, n}-\tilde{a}_{1, n}^{\dagger}\right) \sin n \sigma \\
\pi_{\phi_{1}} & =\sum_{n=1}^{\infty} \sqrt{\frac{\omega_{1, n}}{\pi}}\left(\tilde{a}_{1, n}+a_{1, n}^{\dagger}\right) \sin n \sigma
\end{aligned}
$$




$$
\begin{aligned}
\phi_{2} & =\sum_{n=1}^{\infty} \frac{i}{\sqrt{\pi \omega_{2, n}}}\left(a_{2, n}-\tilde{a}_{2, n}^{\dagger}\right) \sin \left(n-\frac{1}{2}\right) \sigma \\
\pi_{\phi_{2}} & =\sum_{n=1}^{\infty} \sqrt{\frac{\omega_{2, n}}{\pi}}\left(\tilde{a}_{2, n}+a_{2, n}^{\dagger}\right) \sin \left(n-\frac{1}{2}\right) \sigma
\end{aligned}
$$

where

$$
\omega_{1, n}=\sqrt{n^{2}+m^{2}}, \quad \omega_{2, n}=\sqrt{\left(n-\frac{1}{2}\right)^{2}+m^{2}} .
$$

Imposing (4.6), we infer that the bosonic oscillators obey (4.5). The bosonic hamiltonian is given by

$$
H_{B}=\sum_{n=1}^{\infty} \omega_{1, n}\left(a_{1, n}^{\dagger} a_{1, n}+\tilde{a}_{1, n}^{\dagger} \tilde{a}_{1, n}+1\right)+\sum_{n=1}^{\infty} \omega_{2, n}\left(a_{2, n}^{\dagger} a_{2, n}+\tilde{a}_{2, n}^{\dagger} \tilde{a}_{2, n}+1\right) .
$$

Now let us consider fermions. The boundary conditions imposed by supersymmetry are

$$
b_{1}(0)=b_{1}(\pi)=b_{2}(0)=c_{2}(\pi)=0,
$$

which, when combined with the EOM's, give the following boundary conditions for $b$ and $c$ fields separately:

$$
\begin{aligned}
& b_{1}(0)=b_{1}(\pi)=0, \quad b_{2}(0)=b_{2}^{\prime}(\pi)=0, \\
& \left\{\begin{array}{l}
c_{1}^{\prime}(0)-i m \bar{c}_{2}(0)=c_{2}^{\prime}(0)-i m \bar{c}_{1}(0)=0 \\
c_{1}^{\prime}(\pi)=c_{2}(\pi)=0
\end{array}\right.
\end{aligned}
$$

The mode expansions are

$$
\begin{aligned}
b_{1}= & \frac{i}{\sqrt{\pi}} \sum_{n=1}^{\infty} \frac{k_{1, n}-i m}{\omega_{1, n}}\left(\alpha_{n}-\tilde{\alpha}_{n}^{\dagger}\right) \sin k_{1, n} \sigma, \\
b_{2}= & \frac{1}{\sqrt{\pi}} \sum_{n=1}^{\infty} \frac{k_{2, n}+i m}{\omega_{2, n}}\left(\tilde{\beta}_{n}-\beta_{n}^{\dagger}\right) \sin k_{2, n} \sigma, \\
c_{1}= & \frac{1}{\sqrt{\pi}} \sum_{n=1}^{\infty} \frac{k_{1, n}}{k_{1, n}+i m}\left(\alpha_{n}+\tilde{\alpha}_{n}^{\dagger}\right) \cos k_{1, n} \sigma- \\
& -\frac{1}{\sqrt{\pi}} \sum_{n=1}^{\infty} \frac{m}{k_{2, n}+i m}\left(\beta_{n}+\tilde{\beta}_{n}^{\dagger}\right) \sin k_{2, n} \sigma+\lambda \cosh m(\pi-\sigma), \\
c_{2}= & \frac{i}{\sqrt{\pi}} \sum_{n=1}^{\infty} \frac{m}{k_{1, n}-i m}\left(\tilde{\alpha}_{n}+\alpha_{n}^{\dagger}\right) \sin k_{1, n} \sigma- \\
& -\frac{i}{\sqrt{\pi}} \sum_{n=1}^{\infty} \frac{k_{2, n}}{k_{2, n}-i m}\left(\tilde{\beta}_{n}+\beta_{n}^{\dagger}\right) \cos k_{2, n} \sigma-i \lambda^{\dagger} \sinh m(\pi-\sigma),
\end{aligned}
$$

where

$$
k_{1, n}=n, \quad k_{2, n}=n-\frac{1}{2} .
$$


Imposing canonical commutation relations on the fields implies the following commutation relations for the oscillators:

$$
\begin{aligned}
\left\{\alpha_{n}, \alpha_{n^{\prime}}^{\dagger}\right\}=\left\{\tilde{\alpha}_{n}, \tilde{\alpha}_{n^{\prime}}^{\dagger}\right\}=\left\{\beta_{n}, \beta_{n^{\prime}}^{\dagger}\right\}=\left\{\tilde{\beta}_{n}, \tilde{\beta}_{n^{\prime}}^{\dagger}\right\} & =\delta_{n n^{\prime}}, \\
\left\{\lambda, \lambda^{\dagger}\right\} & =\frac{m}{\sinh 2 m \pi} .
\end{aligned}
$$

All other anti-commutators vanish.

In view of the commutation relations for $\lambda$ and $\lambda^{\dagger}$, we set

$$
\lambda=\sqrt{\frac{m}{\sinh 2 m \pi}} \zeta
$$

so that

$$
\{\zeta, \zeta\}=0, \quad\left\{\zeta, \zeta^{\dagger}\right\}=1
$$

The fermionic hamiltonian has the form

$$
\begin{aligned}
H_{F} & =\int_{0}^{\pi} \mathrm{d} \sigma\left[\left(i c_{1}^{\prime}+m \bar{c}_{2}\right) \bar{b}_{1}+\left(i c_{2}^{\prime}+m \bar{c}_{1}\right) \bar{b}_{2}+\text { h.c. }\right] \\
& =\sum_{n=1}^{\infty} \omega_{1, n}\left(\alpha_{n}^{\dagger} \alpha_{n}+\tilde{\alpha}_{n}^{\dagger} \tilde{\alpha}_{n}-1\right)+\sum_{n=1}^{\infty} \omega_{2, n}\left(\beta_{n}^{\dagger} \beta_{n}+\tilde{\beta}_{n}^{\dagger} \tilde{\beta}_{n}-1\right) .
\end{aligned}
$$

As before the bosonic zero-point energy is canceled by the fermionic zero-point energy. There are two zero-energy states:

$$
|0\rangle \quad \text { and } \quad \zeta^{\dagger}|0\rangle
$$

Again it can be shown that they are annihilated by the supercharge. Therefore both ground states are supersymmetric.

\section{Topological correlators in the LG model $W=x y$}

\subsection{Topological B-twist}

The Landau-Ginzburg model admits a topological twist to yield the so-called B-model. This topological twist turns the world-sheet spinor fields $\psi$ and $\bar{\psi}$ of the original LG model into a pair of sections of the pullback bundle $\Phi^{*}\left(T^{0,1} X\right)$, which we denote by $\eta, \theta$, and a world-sheet one-form $\rho$ with values in $\Phi^{*}\left(T^{1,0} X\right)$. The BRST transformations of the twisted fields are

$$
\begin{aligned}
\delta \phi^{i} & =0, \\
\delta \phi^{i} & =\bar{\epsilon} \eta^{\bar{i}}, \\
\delta \eta^{\bar{i}} & =0, \\
\delta \theta^{\bar{i}} & =\bar{\epsilon} \partial^{\bar{i}} W, \\
\delta \rho^{i} & =i \bar{\epsilon} \mathrm{d} \phi^{i} .
\end{aligned}
$$


To make connection with the fields in the original Landau-Ginzburg theory, we note that $\eta$ and $\theta$ are the twisted versions of $\bar{b}$ and $\bar{c}$ respectively, while $\rho$ comes from $b$ and $c$. We also adopt the common notation $\theta_{i}=g_{i \bar{j}} \theta^{\bar{j}}=\theta^{\bar{i}}$.

The local physical observables are in one-to-one correspondence with the BRST-cohomology, i.e. local quantities which are BRST invariant but not BRST exact. It is easy to see from the above BRST transformations that in the bulk the physical observables correspond to holomorphic functions of $\phi$ modulo $\mathrm{d} W(v)$, where $v$ is an arbitrary holomorphic vector field. There are no additional local observables from the fermionic fields, as long as $W$ is nontrivial. In particular, when $X \simeq \mathbb{C}^{n}$, the space of bulk observables is $\mathbb{C}\left[x_{1}, \ldots, x_{n}\right] / I$, where $I$ is the ideal generated by the first partial derivatives of $W$. In the boundary sector, where $W$ is constrained to be constant, additional observables will arise from the $\theta$ fields.

We now specialize to the D0 and D2 branes studied above.

\subsection{Boundary observables associated with the D0-brane}

We consider the boundary component which is mapped to the D0 brane located at $\phi_{1}=$ $\phi_{2}=0$. The boundary conditions require, among other things, that

$$
\phi_{1}=\phi_{2}=\eta^{\overline{1}}=\eta^{\overline{2}}=0 \quad \text { at the boundary . }
$$

Therefore boundary observables can only come from the $\theta$ fields. From the BRST transformation

$$
\delta \theta_{1}=2 \bar{\epsilon} m \phi_{2}, \quad \delta \theta_{2}=2 \bar{\epsilon} m \phi_{1}
$$

one sees immediately that both $\theta_{1}$ and $\theta_{2}$ are BRST invariant on the boundary. Let us denote the restriction of $\theta$ to the boundary by the same letter $\theta$. Thus the ring of boundary observables is generated by $\theta_{1}$ and $\theta_{2}$.

\subsection{Boundary observables associated with the D2-brane}

Without loss of generality, we may assume that the D2 brane sits at the locus $\phi_{1}=0$. The relevant boundary conditions read

$$
\phi_{1}=\partial_{\sigma} \phi_{2}=\eta^{\overline{1}}=\theta_{2}=0 \quad \text { at the boundary . }
$$

From this one sees that $\theta_{2}$ no longer gives rise to a boundary degree of freedom. Also, since $\phi_{2}$ is not constrained to vanish on the boundary, $\theta_{1}$ is no longer BRST invariant. Thus there are no boundary observables (except for the identity operator) associated with the D2 brane.

\subsection{The boundary operator product algebra}

First let us compute topological correlators for strings connecting a brane with itself. Since in the D2-D2 case there is only the vacuum state, the problem is non-trivial only in the D0-D0 case. 


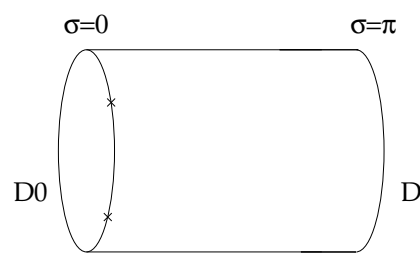

(a)

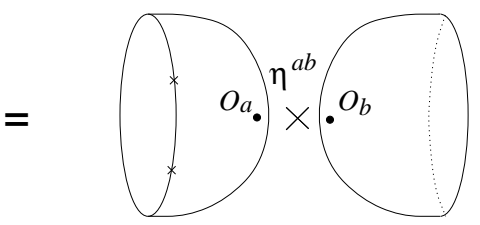

(b)

Figure 1.

In order to compute disk correlators with products of $\theta_{1}$ and $\theta_{2}$ inserted on the circumference, we proceed as in ref. [16]. We start with a world-sheet diagram which has the topology of a cylinder, with the D0 and D2 boundary conditions imposed on the two boundary circles (c.f. figure ??). On the D0 boundary there can be operator insertions. Viewed in the open-string channel, this world-sheet diagram computes the one-loop amplitude

$$
\left\langle\mathcal{B}_{1} \mathcal{B}_{1} \cdots \mathcal{B}_{r}\right\rangle_{\text {cyl }}=\operatorname{Tr}\left[(-)^{F} e^{-\epsilon H} \mathcal{B}_{1} \mathcal{B}_{2} \cdots \mathcal{B}_{r}\right]
$$

where the $\mathcal{B}$ 's are operators inserted at the D0 boundary. The trace on the r.h.s. can be reduced to the Hilbert space of open-string zero modes by the standard argument. In the specific case at hand, the zero mode space is spanned by $|0\rangle$ and $\zeta^{\dagger}|0\rangle$ as described in section 4.5, and one easily obtains

$$
\begin{aligned}
\langle 1\rangle_{\mathrm{cyl}} & =0 \\
\left\langle\theta_{i}\right\rangle_{\mathrm{cyl}} & =0 \\
\left\langle\theta_{1} \theta_{2}\right\rangle_{\mathrm{cyl}} & =-\left\langle\theta_{2} \theta_{1}\right\rangle_{\mathrm{cyl}}=-\frac{i m}{2} .
\end{aligned}
$$

More generally, one can compute

$$
\begin{aligned}
\left\langle\left(\theta_{1} \theta_{2}+\theta_{2} \theta_{1}\right) \cdot \mathcal{B}\right\rangle_{\mathrm{cyl}} & =-\frac{i m}{2}\langle\mathcal{B}\rangle_{\mathrm{cyl}} \quad \forall \mathcal{B}, \\
\left\langle\theta_{i}^{2} \cdot \mathcal{B}\right\rangle_{\mathrm{cyl}} & =0 \quad \forall \mathcal{B}, \forall i .
\end{aligned}
$$

From (5.2) and (5.3) one deduces the relations in the boundary operator product algebra for the D0-brane:

$$
\begin{array}{r}
\theta_{1} \cdot \theta_{1}=\theta_{2} \cdot \theta_{2}=0, \\
\theta_{1} \cdot \theta_{2}+\theta_{2} \cdot \theta_{1}=-\frac{i m}{2} .
\end{array}
$$

This is the Clifford algebra with two generators corresponding to the quadratic form

$$
-\frac{i}{2}\left(\begin{array}{cc}
0 & m \\
m & 0
\end{array}\right) .
$$

Up to a numerical factor, this matrix is the hessian of $W$ in the basis $\theta_{1}, \theta_{2}$. Thus one can state the result more invariantly by saying that the boundary operator product algebra for the D0-brane is the Clifford algebra $\mathrm{Cl}(V, Q)$, where $V$ is target space of our LG model, and $Q$ is the quadratic form given by the hessian of $W$. 
Topological correlators on the disk can be inferred from the computation on the cylinder using factorization in the closed string channel [28]. Namely, we insert a complete set of states in the closed string channel (cf. figure ??b) and rewrite the cylinder amplitude as

$$
\left\langle\mathcal{B} \cdot O_{a}\right\rangle_{\mathrm{D} 0} \cdot \eta^{a b} \cdot\left\langle O_{b}\right\rangle_{\mathrm{D} 2},
$$

where $O_{a}$ 's form a complete set of bulk operators, and $\eta$ is the (inverse) metric on the space of bulk operators defined via topological correlators on the sphere. The relative normalization is fixed by demanding the following relation for the D2-D2 cylinder amplitude

$$
\langle 1\rangle_{\mathrm{D} 2-\mathrm{D} 2}=1 \text {. }
$$

In our case, the only bulk operator is the identity, therefore all disk correlators for the D0-brane simply coincide with the cylinder correlators.

Besides the algebra structure, another important datum is a non-degenerate inner product on the space of endomorphisms. This inner product is determined by the two-point disk correlator and makes the endomorphism algebra into a (non-commutative) graded Frobenius algebra. In our case the only non-vanishing inner products are

$$
\left\langle\theta_{1} \theta_{2}, 1\right\rangle=\left\langle 1, \theta_{1} \theta_{2}\right\rangle=\left\langle\theta_{1}, \theta_{2}\right\rangle=-\left\langle\theta_{2}, \theta_{1}\right\rangle=-\frac{i m}{2} .
$$

Note that the bilinear form corresponding to this product is even. In contrast, in the model $W=z^{2}$ the bilinear form is odd and given by

$$
\langle 1, \theta\rangle=\langle\theta, 1\rangle=1 .
$$

So far we have determined the endomorphism algebra of the D0-brane (it is isomorphic to $\mathrm{Cl}(2, \mathbb{C})$ ) and the D2-brane (it is isomorphic to $\mathbb{C}$ ). Now we turn to the computation of compositions of morphisms between different branes.

We begin with the case when both branes are D2-branes. Let us denote the D2-brane given by the equation $\Phi_{1}=0\left(\operatorname{resp} . \Phi_{2}=0\right)$ by $Y_{2}\left(\right.$ resp. $\left.Y_{1}\right)$. It was shown in the previous section that the vector space $\operatorname{Mor}\left(Y_{i}, Y_{j}\right)$ is one-dimensional for all $i$ and $j$. When $i=j$, this space is even, but for $i \neq j$ there is no canonical choice for the R-charge. In other words, for $i \neq j$ the "vacuum" vector spanning $\operatorname{Mor}\left(Y_{i}, Y_{j}\right)$ can equally well be regarded as even or odd. For reasons which will become clear later, we define $\operatorname{Mor}\left(Y_{1}, Y_{2}\right)$ to be purely odd; since $\left.\operatorname{Mor}\left(Y_{2}, Y_{1}\right)\right)$ is dual to $\operatorname{Mor}\left(Y_{1}, Y_{2}\right)$, it is also purely odd, while $\operatorname{Mor}\left(Y_{1}, Y_{2}[1]\right)$ is purely even. Here $Y_{2}[1]$ denotes the shift of $Y_{2}$.

Let $\gamma_{12}$ and $\gamma_{21}$ be generators of $\operatorname{Mor}\left(Y_{1}, Y_{2}\right)$ and $\operatorname{Mor}\left(Y_{2}, Y_{1}\right)$, respectively. Since the endomorphism algebra of a D2-brane is spanned by the identity morphism, we only need to determine if $\gamma_{12} \cdot \gamma_{21}$ is zero or not. This product is evaluated by the disk amplitude with two insertions of boundary-changing operators. By conformal invariance, this is the same as the vacuum-vacuum transition amplitude for open strings stretched between $Y_{1}$ and $Y_{2}$. Since there are no fermionic zero modes in this case, this amplitude is non-zero. This means that $\gamma_{12} \cdot \gamma_{21}=c \cdot i d$ with $c \neq 0$.

This trivial computation implies that the even generator of $\operatorname{Mor}\left(Y_{1}, Y_{2}[1]\right)$ is an isomorphism. In physical language, $Y_{1}$ is isomorphic to the anti-brane of $Y_{2}$. 
There remain compositions of morphisms involving both D2-branes and the D0-brane. In view of the previous paragraph, it is sufficient to consider morphisms between $Y_{1}$ and the D0-brane. No new computations are actually required, the result being fixed by general properties of topological string theory [22, 23]. First of all, we note that by cyclic symmetry of topological correlators computing compositions of morphisms from D2 to D0 and back (or the other way around) is equivalent to computing how the endomorphism algebra of the D0-brane acts on the space of morphisms from D2 to D0. In more detail, we have a non-degenerate pairing

$$
\operatorname{Mor}\left(Y_{1}, D 0\right) \times \operatorname{Mor}\left(D 0, Y_{1}\right) \rightarrow \mathbb{C}
$$

given by the path-integral on an infinite strip. (This paring is odd in our case, because there is a single fermionic zero mode.) Similarly, we have an even non-degenerate pairing

$$
\operatorname{Mor}(D 0, D 0) \times \operatorname{Mor}(D 0, D 0) \rightarrow \mathbb{C} .
$$

Thus computing the product map

$$
\operatorname{Mor}\left(D 0, Y_{1}\right) \times \operatorname{Mor}\left(Y_{1}, D 0\right) \rightarrow \operatorname{Mor}(D 0, D 0)
$$

is the same as computing the map

$$
\operatorname{Mor}\left(Y_{1}, D 0\right) \times \operatorname{Mor}(D 0, D 0) \rightarrow \operatorname{Mor}\left(Y_{1}, D 0\right)
$$

Furthermore, in our case $\operatorname{Mor}(D 0, D 0)$ is isomorphic to $\mathrm{Cl}(2, \mathbb{C})$, and we know from the previous section that $\operatorname{Mor}\left(Y_{1}, D 0\right)$ is two-dimensional. The $\mathbb{Z}_{2}$ graded algebra $\mathrm{Cl}(2, \mathbb{C})$ has a unique representation on $\mathbb{C}^{2}$, up to a flip of parity (up to isomorphism, it is given by any two Pauli matrices). Since in string theory the parity of morphisms is not canonically fixed anyway, we conclude that the module structure of $\operatorname{Mor}\left(Y_{1}, D 0\right)$ is completely determined, up to the unavoidable ambiguity in the overall parity. This in turn determines the composition of morphisms going from D0 to D2 and back.

\section{B-branes in general massive LG models}

\subsection{Generalities}

We now turn to massive Landau-Ginzburg models which involve more than two fields. Without loss of generality, we may assume that the superpotential on $\mathbb{C}^{n}$ is given by

$$
W=z_{1}^{2}+\cdots+z_{n}^{2}
$$

We can construct examples of B-branes in this LG model for any $n$, using the results of the previous section. For $n=2 k, k \in \mathbb{Z}$, we consider an equivalent superpotential

$$
W=z_{1} z_{2}+z_{3} z_{4}+\cdots+z_{2 k-1} z_{2 k}
$$


Since it is a sum of $k$ copies of the superpotential $W=x y$, we can construct a B-type boundary condition by picking $k$ arbitrary B-type boundary conditions for the latter model, and tensoring them. For example, if we take all boundary conditions to be D0-branes, the tensor product state will also be a D0-brane, and its endomorphism algebra will be $\mathrm{Cl}(2 k, \mathbb{C})$. If we take all boundary conditions to be $\mathrm{D} 2$-branes, the tensor product boundary state will be a $\mathrm{D}(2 k)$-brane, and its endomorphism algebra will be $\mathbb{C}$.

Similarly, for $n=2 k+1$ we consider an equivalent superpotential

$$
W=z_{1} z_{2}+z_{3} z_{4}+\cdots+z_{2 k-1} z_{2 k}+z_{2 k+1}^{2} .
$$

Clearly, B-branes for this LG model can be constructed by taking tensor product of $k$ boundary states for the LG model with $W=x y$ and a boundary state for the LG model with $W=z^{2}$. In this way one obtains B-branes of dimension up to $2 k$. It is easy to see that the endomorphism algebra of the D0-brane will be isomorphic to $\mathrm{Cl}(2 k+1, \mathbb{C})$, while the endomorphism algebra of the $\mathrm{D}(2 k)$-brane will be isomorphic to $\mathrm{Cl}(1, \mathbb{C})$.

More generally, one can explicitly construct all B-branes which correspond to linear subspaces of the critical level set $W=0$. Since $W$ is quadratic, these are the same as linear subspaces isotropic with respect to the bilinear form $Q$. Classification of such isotropic subspaces is well known [29]. The maximal dimension of an isotropic subspace is $[n / 2]$. For $n$ odd, there is a single irreducible family of isotropic subspaces of maximal dimension parametrized by $(n-1)(n+1) / 8$ parameters. For $n$ even, there are two irreducible families of isotropic subspaces of maximal dimension parametrized by $n(n-2) / 8$ parameters. Any isotropic subspace lies in one of the maximal isotropic subspaces. It is straightforward to compute morphisms and their compositions (i.e the spectrum and topological correlators) between all linear B-branes. In the next subsection we discuss in some detail the results for the case $n=3$, when the LG superpotential has the form $W=x y+z^{2}$. Then we will describe the general case.

Note that in principle there could also be B-branes corresponding to non-linear boundary conditions (e.g. non-linear submanifolds of the quadric $W=0$ ). Such B-branes are hard to study directly. In what follows we shall focus on linear boundary conditions.

\subsection{The LG model with the superpotential $W=x y+z^{2}$}

Maximal isotropic linear subspaces on the quadric surface $W=0$ are complex lines, and there is a single irreducible family of them. This family is parametrized by $\mathbb{C P}^{1}$ as follows:

$$
\mu x+\nu z=0, \quad \mu z-\nu y=0,
$$

where $[\mu: \nu]$ are homogeneous coordinates on $\mathbb{C P}^{1}$. Any two distinct lines in the family intersect at a single point $(x=y=z=0)$.

Using a linear change of basis in the target space which preserves $W$, one can always map any line in the above family to the line $x=z=0$. For the brane $x=z=0$ we already know that the endomorphism algebra is isomorphic to $\mathrm{Cl}(1, \mathbb{C})$, and since linear changes of variables preserving the superpotential are invariances of the topological LG model, we conclude that the same is true for any D2-brane in the above family. 
Next we consider morphisms between different lines in the family. Clearly, there are no bosonic zero modes, so the space of morphisms will be spanned by the "vacuum" state and its fermionic excitations with zero energy. As remarked in the previous section, only some components of $\theta$ have a chance to be BRST-non-trivial boundary observables. Thus all BRST-invariant states can be obtained by acting by some components of $\theta$ on the vacuum state.

Let $V \simeq \mathbb{C}^{3}$ be the target space of our LG model, and let $U_{1}$ and $U_{2}$ be two distinct lines in $V$ isotropic with respect to the quadratic form $W$. The corresponding B-branes will be denoted $Y_{1}$ and $Y_{2}$. Let us look at the $\theta$-field restricted to the boundary of the world-sheet which is mapped to $U_{1}$. We can regard $\theta_{i}$ as basis elements of $V$. BRST transformations are

$$
\delta \theta_{i}=Q_{i j} \phi^{j}
$$

On the boundary the vector with components $\phi^{j}$ can be an arbitrary element of $U_{1}$. It follows that BRST-invariant components of $\theta$ must be orthogonal to $U_{1}$ with respect to the form $Q$. We denote the orthogonal subspace by $U_{1}^{\perp}$. Of course, since $U_{1}$ is isotropic, we have an inclusion $U_{1} \subset U_{1}^{\perp}$. Similarly, BRST-invariant fermionic fields on the $U_{2}$-boundary are parametrized by elements of $U_{2}^{\perp} \supset U_{2}$. The total space of BRST-invariant fermionic fields is

$$
U_{1}^{\perp} \oplus U_{2}^{\perp}=V .
$$

However, not all of these are non-zero. Neumann boundary conditions plus supersymmetry imply that the components of $\theta$ along $U_{1} \oplus U_{2}$ vanish. Thus non-trivial BRST invariant fermionic zero modes are parametrized by elements of the quotient space

$$
\frac{V}{\left(U_{1} \oplus U_{2}\right)} \text {. }
$$

This space is one-dimensional. Thus there is a single fermionic zero mode, and the space of morphisms between two different lines is isomorphic to its exterior algebra (as a $\mathbb{Z}_{2^{-}}$ graded vector space). That is, $\operatorname{Mor}\left(Y_{1}, Y_{2}\right)$ has one-dimensional even subspace, and onedimensional odd subspace.

Composition of morphisms between two distinct lines is fixed by consistency considerations. If $Y_{1}$ and $Y_{2}$ are any two lines, then $\operatorname{Mor}\left(Y_{1}, Y_{2}\right)$ must be a left module over $\operatorname{Mor}\left(Y_{1}, Y_{1}\right) \simeq \mathrm{Cl}(1, \mathbb{C})$ and right module over $\operatorname{Mor}\left(Y_{2}, Y_{2}\right) \simeq \mathrm{Cl}(1, \mathbb{C})$. There is only one such module of dimension two: the Clifford algebra itself, regarded as a bi-module over itself. Together with various parings given by the 2-point correlators, this fixes the structure of correlators involving any two D2-branes. In particular, it is easy to see that the element in $\operatorname{Mor}\left(Y_{1}, Y_{2}\right)$ corresponding to the identity element in $\mathrm{Cl}(1, \mathbb{C})$ is invertible. This means that any two lines give isomorphic objects in the category of B-branes.

Similar arguments can be used to determine boundary correlators involving both D2 and D0. As explained above, the endomorphism algebra of the D0-brane is isomorphic to $\mathrm{Cl}(V, Q) \simeq \mathrm{Cl}(3, \mathbb{C})$. As for the space of morphisms between a D2-brane and D0-brane, it is 4-dimensional, with two-dimensional even subspace and two-dimensional odd subspace. Indeed, since all D2-branes are isomorphic, it is sufficient to consider morphisms from the 
D2-brane $x=z=0$. This D2-brane is the tensor product of the D2-brane in the LG model $W=x y$ and the D0-brane in the LG model $W=z^{2}$. Hence the computation of the space of morphisms and their compositions is reduced to the one we have performed in the previous section.

\subsection{The LG model with the superpotential $W=z_{1}^{2}+\cdots+z_{n}^{2}$}

The above arguments can be easily generalized to arbitrary $n$. We shall consider only linear boundary conditions. Let the target space be $V \simeq \mathbb{C}^{n}$, let $W$ be a non-degenerate quadratic function on $V$, and let $Q \in \operatorname{Sym}^{2}\left(V^{*}\right)$ be its hessian. A B-brane is a linear subspace $U \subset V$ which is isotropic with respect to $Q$. As mentioned above, $k=\operatorname{dim}_{\mathbb{C}} U$ is less or equal to $[n / 2]$.

Using linear changes of variables, we can bring $W$ to the standard form, and $U$ to the subspace given by $z_{1}=\cdots=z_{n-k}=0$. Such a $\mathrm{D}(2 \mathrm{k})$-brane is a tensor product of $k$ copies of D2-branes in the model $W=x y,[n / 2]-k$ copies of the D0-brane in the model $W=x y$, and, for $n$ odd, one copy of a D0-brane in the model $W=z^{2}$. It follows that the space of endomorphisms has dimension

$$
\operatorname{dim}_{\mathbb{C}} \operatorname{End}(D(2 k))=2^{n-2 k},
$$

and is isomorphic as a $\mathbb{Z}_{2}$-graded algebra to the Clifford algebra with $n-2 k$ generators. In particular, the algebra of endomorphisms of a D-brane of maximal possible dimension is isomorphic to $\mathbb{C}$ or $\mathrm{Cl}(1, \mathbb{C})$ depending on whether $n$ is even or odd, while the the endomorphism algebra of the D0-brane is isomorphic to $\mathrm{Cl}(V, Q) \simeq \mathrm{Cl}(n, \mathbb{C})$.

Next let us discuss morphisms between two different B-branes. Let $U_{1}$ and $U_{2}$ be isotropic linear subspaces corresponding to B-branes $Y_{1}$ and $Y_{2}$. The same arguments as in the previous subsection tell us that the space of fermionic zero modes can be identified with

$$
\frac{\left(U_{1}^{\perp} \oplus U_{2}^{\perp}\right)}{\left(U_{1} \oplus U_{2}\right)}
$$

The space of morphisms is isomorphic as a graded vector space to the exterior algebra of this vector space (up to an overall flip of parity). It is easy to see that the dimension of the space of zero modes is given by $n-k_{1}-k_{2}$, where $k_{i}=\operatorname{dim} U_{i}$. Therefore the dimension of the space of morphisms is given by

$$
2^{n-k_{1}-k_{2}}
$$

In particular, in the case $U_{1}=U_{2}$ we recover the result eq. 6.3. obtained by other means.

Let us give a few examples. First, let $n$ be even, and $U_{1}$ and $U_{2}$ be distinct maximal isotropic subspaces. Then $U_{i}^{\perp}=U_{i}$ for $i=1,2$, and there are no zero modes. This means that the space of morphisms between any two maximal isotropic subspaces is onedimensional. As usual, the R-charge assignment is ambiguous, but it is natural to require the R-charge to vary continuously as one varies $U_{i}$. Since in the case $U_{1}=U_{2}$ the space of endomorphisms is even and isomorphic to $\mathbb{C}$, this implies that for any two maximal isotropic subspaces in the same irreducible family the space of morphisms is even and isomorphic 
to $\mathbb{C}$. We will fix the remaining ambiguity by saying that the space of morphisms between two maximal isotropic subspaces in different irreducible families is odd. The reason for such a convention will be explained in the next section.

The fact that for even $n$ there are no fermionic zero modes for open strings connecting two maximal isotropic subspaces implies that the vacuum-vacuum transition amplitude is non-zero in this sector. This is equivalent to saying that the composition of non-zero morphisms between two maximal B-branes is a non-zero multiple of the identity endomorphism. If these two B-branes are in the same irreducible family, this means that they represent isomorphic objects in the category; if they are in different irreducible families, then the interpretation is that they are isomorphic up to a shift.

If $n$ is odd, and $U_{1}$ and $U_{2}$ are maximal linear subspaces, then there is a single fermionic zero mode. Thus $\operatorname{Mor}\left(Y_{1}, Y_{2}\right)$ is two-dimensional, with one-dimensional even and onedimensional odd subspaces. The composition of morphisms going between two maximal B-branes is fixed by consistency requirements. Namely, the space of morphisms must be a graded bi-module over $\mathrm{Cl}(1, \mathbb{C})$ (the endomorphism algebra of a single B-brane), and there is only one such graded bi-module of dimension two: $\mathrm{Cl}(1, \mathbb{C})$ itself. Furthermore, there is an odd non-degenerate pairing

$$
\operatorname{Mor}\left(Y_{1}, Y_{2}\right) \times \operatorname{Mor}\left(Y_{2}, Y_{1}\right) \rightarrow \mathbb{C},
$$

which is invariant with respect to both actions of $\mathrm{Cl}(1, \mathbb{C})$ in an obvious sense. Up to isomorphism, there is only one such pairing, namely

$$
\langle a, b\rangle=\operatorname{tr}(a b)
$$

where $t r$ is defined by

$$
\operatorname{tr}(1)=0, \quad \operatorname{tr}(\theta)=1 .
$$

Together with the module structure of $\operatorname{Mor}\left(Y_{1}, Y_{2}\right)$, this pairing determines the composition of morphisms going between any two lines. As in the case $W=x y+z^{2}$, it is easy to see that the morphism corresponding to the identity element of $\mathrm{Cl}(1, \mathbb{C})$ is invertible, and therefore any two maximal B-branes are isomorphic.

Our third example is the case $U_{1}=U_{2}=0$, that is, the case of the D0-brane. The space of zero modes coincides with $V$, and the space of endomorphism is isomorphic to $\wedge^{*} V$ as a $\mathbb{Z}_{2}$-graded vector space ( $V$ is regarded as odd). This agrees with an independent argument of subsection 6.1. There we also showed that the algebra of endomorphisms is isomorphic to $\mathrm{Cl}(V, Q)$.

Our fourth and final example is the case when $U_{1}$ is an arbitrary isotropic subspace of dimension $k \leq[n / 2]$, and $U_{2}=0$. In other words, the second brane is the D0-brane. Then the space of zero modes is $V / U_{1}$. Its dimension is $n-k$, and therefore the space of open strings stretched between a maximal linear subspace and the D0-brane has dimension $2^{n-k}$. The space of morphisms has the structure of a graded module over the endomorphism algebra of the $\mathrm{D} 0$-brane, which is isomorphic to $\mathrm{Cl}(V, Q)$. If we neglect the grading, there is a unique such module, which is a sum of $2^{[n / 2]-k}$ irreducible (spinor) modules. 


\section{B-branes and twisted complexes}

\subsection{Kontsevich's proposal ${ }^{5}$}

Let us recall how to define the derived category of coherent sheaves on a smooth affine variety $X$ following ref. [30]. Let $\operatorname{Coh}(X)$ be the category of coherent sheaves on $X$, or equivalently the category of finite modules over the coordinate $\operatorname{ring} \mathcal{O}_{X}$ of $X$. We define $C(X)$ to be a category whose objects are bounded $\mathbb{Z}$-graded complexes of projective objects of $\operatorname{Coh}(X)$. Equivalently, we can think about the coordinate ring of $X$ as a differential graded algebra (dg-algebra) which is concentrated in degree zero and has a trivial differential; then objects of $C(X)$ are differential graded modules (dg-modules) over this dg-algebra such that all homogeneous components are projective $\mathcal{O}_{X}$-modules, and all but a finite number of homogeneous components are trivial. Morphisms in $C(X)$ are morphisms of these dg-modules regarded simply as $\mathcal{O}_{X}$-modules (i.e. morphisms do not necessarily preserve the grading or respect the differentials). Groups of morphisms in the category $C(X)$ are naturally $\mathbb{Z}$-graded and have a natural differential of degree 1 . For example, closed morphisms of degree 0 in the category $C(X)$ are ordinary morphisms of complexes (the ones which preserve the grading and commute with the differentials), while exact morphisms of degree 0 are morphisms of complexes which are homotopic to zero. Thus $C(X)$ is a dg-category.

There is a general way to make a triangulated category out of any dg-category [30. One takes the category of "twisted objects" of the dg-category, which is again a dg-category, and then passes to degree- 0 homology, i.e. replaces groups of morphisms with their degree- 0 homology. In the present case, since we are working with complexes of projective modules, it is not necessary to consider twisted objects, and one can simply apply the functor $H^{0}$ to $C(X)$. The resulting triangulated category is simply the homotopy category of bounded complexes of projective $\mathcal{O}_{X}$ modules, and it is well known that it is equivalent to the bounded derived category of $\operatorname{Coh}(X)$ (see e.g. ref. [31]). Alternatively, one can apply to $C(X)$ the functor $H^{*}$. This gives a graded category which is the completion of $D^{b}(\operatorname{Coh}(X))$ with respect to the shift functor. As discussed in section 2 2 , the latter alternative conforms better to physical conventions.

Now we can formulate Kontsevich's proposal rather simply. Let $X$ be a smooth affine variety, and $W$ be a holomorphic function on $X$ (the superpotential), whose critical set is compact. Let $W_{0} \in \mathbb{C}$ be a critical value of $W$. First, since in the presence of the superpotential morphisms between B-branes are $\mathbb{Z}_{2}$-graded, we will have to use $\mathbb{Z}_{2}$-graded complexes in order to construct the analogue of $C(X)$. Second, we deform our $\mathbb{Z}_{2}$-graded complexes of projective modules by asking that the composition of two successive morphisms be equal to $W-W_{0}$, instead of zero. Thus objects of the deformed category $C\left(X, W, W_{0}\right)$ are pairs of finitely generated projective $\mathcal{O}_{X}$-modules $E_{0}, E_{1}$ and morphisms $d_{0}: E_{0} \rightarrow E_{1}$ and $d_{1}: E_{1} \rightarrow E_{0}$ such that

$$
d_{1} d_{0}=W-W_{0}, \quad d_{0} d_{1}=W-W_{0} .
$$

\footnotetext{
${ }^{5}$ The content of this subsection was explained to us by Maxim Kontsevich.
} 
We can regard the pair $\left(E_{0}, E_{1}\right)$ as a $\mathbb{Z}_{2}$-graded $\mathcal{O}_{X}$-module, and $\left(d_{0}, d_{1}\right)$ as an odd endomorphism $d_{E}$ of this module whose square is $W-W_{0}$ ("twisted differential"). Morphisms in this category are defined as (ungraded) morphisms of the corresponding $\mathcal{O}_{X}$-modules. They have a natural $\mathbb{Z}_{2}$-grading, and a natural differential. The differential on $\operatorname{Mor}(E, F)$ is defined as

$$
D \phi=\phi d_{E}+(-1)^{\operatorname{deg}} \mathrm{d}_{F} \phi .
$$

Here $(-1)^{\operatorname{deg}}: \operatorname{Mor}(E, F) \rightarrow \operatorname{Mor}(E, F)$ acts as 1 on the even component and as -1 on the odd component. It is easy to see that $D: \operatorname{Mor}(E, F) \rightarrow \operatorname{Mor}(E, F)$ is an odd operator whose square is zero. Thus $C\left(X, W, W_{0}\right)$ is a differential $\mathbb{Z}_{2}$-graded category. In what follows the term "dg-category" (resp. "graded category") will refer to a differential $\mathbb{Z}_{2}$-graded category (resp. $\mathbb{Z}_{2}$-graded category), unless specified otherwise.

Applying to $C\left(X, W, W_{0}\right)$ the functor $H^{*}$, we obtain a graded category, which is proposed to be equivalent to the category of B-branes corresponding to the critical value $W_{0}$. One can show that all spaces of morphisms in this category are finite-dimensional, provided the critical set of $W$ is compact.

An unsatisfactory feature of this construction is that one needs to use complexes of projective modules, instead of general complexes. This causes problems if one tries to extend the definition from affine varieties to algebraic ones. There is a way to repair this defect [32], but we will not try to explain this more complicated definition in this paper.

\subsection{A physical derivation of Kontsevich's proposal}

In this subsection we give a physical argument supporting the identification of B-branes with objects of the category $C\left(X, W, W_{0}\right)$. Our argument is modelled on those in refs. [9, 20, 21], where it was explained why complexes of locally free sheaves on a Calabi-Yau manifold can be thought of as B-branes. For our purposes, it is sufficient to consider $\mathbb{Z}_{2^{-}}$ graded complexes. Then the argument of refs. [9, 20, 21] can be summarized as follows. Consider a pair of locally free sheaves (i.e. holomorphic vector bundles) $E_{1}$ and $E_{2}$. We already know that $E_{1}$ and $E_{2}$ can be thought of as B-branes, i.e. as topological boundary conditions for a topological sigma-model. The same goes for $E_{1} \oplus E_{2}$ and $E_{1} \oplus E_{2}$ [1]. (In the physical setting, one has additional data, such as hermitean metrics on $E_{1}$ and $E_{2}$ and compatible connections.) Now we can deform the boundary condition corresponding to $E_{1} \oplus E_{2}[1]$ by adding a boundary term to the action which depends on a pair of sections $F \in \operatorname{Hom}\left(E_{1}, E_{2}\right)$ and $G \in \operatorname{Hom}\left(E_{2}, E_{1}\right)$ (the "tachyons"). In order to preserve BRST invariance, one has to require that $F$ and $G$ be holomorphic, and $F G=0, G F=0$. This deformed boundary condition corresponds to a $\mathbb{Z}_{2}$-graded complex

$$
E_{1} \stackrel{F}{\longrightarrow} E_{2} \stackrel{G}{\longrightarrow} E_{1}
$$

One expects (although there is no iron-clad argument) that any B-brane is isomorphic to a B-brane of this kind. This provides a physical explanation for the relation between complexes of locally free sheaves on a Calabi-Yau and B-branes. 
In the case of a LG model, locally free coherent sheaves on $X$ are not valid B-branes, because their support is the whole $X$, and $W$ is not constant on $X$. Technically, the problem occurs because the BRST variation of the bulk action contains a non-vanishing $W$-dependent term which is a total derivative on the world-sheet. This is the so-called Warner problem [33]. The sum $E_{1} \oplus E_{2}[1]$ is not a B-brane either. However, we can try to add a boundary term to the action of the sigma-model so that BRST invariance is restored. We take the same term as in the case $W=0$. As in refs. [9, 20, 21], we have two holomorphic sections $F$ and $G$ to play with. As we show below, the condition of BRST-invariance is modified to $F G=W-W_{0}, G F=W-W_{0}$, where $W_{0}$ is a constant. This shows that any object of $C\left(X, W, W_{0}\right)$ corresponds to a B-brane.

Now let us work out the BRST-invariance conditions and show that $F$ and $G$ must satisfy the constraints stated above. For simplicity, let us assume at first that both $E_{1}$ and $E_{2}$ are line bundles. The boundary lagrangian is taken to be

$$
\mathcal{L}_{b}=\frac{i}{2}\left(\bar{\gamma} D_{\tau} \gamma+\psi^{i} \partial_{i} F \gamma+\psi^{\bar{i}} \partial_{\bar{i}} \bar{G} \gamma\right)-\frac{1}{4}(\bar{F} F+\bar{G} G)+\text { h.c. }
$$

Here $\gamma$ is a complex fermion living on the boundary, $\psi^{i}=\psi_{+}^{i}+\psi_{-}^{i}$ is the restriction of a bulk fermionic field to the boundary, and $F=F(\phi), G=G(\phi)$ are holomorphic sections of $\operatorname{Hom}\left(E_{1}, E_{2}\right)$ and $\operatorname{Hom}\left(E_{2}, E_{1}\right)$, respectively. They depend on the fields $\phi^{i}$ restricted to the boundary. The fermion $\gamma$ takes values in $\operatorname{Hom}\left(E_{2}, E_{1}\right)$, and the covariant derivative $D_{\tau}$ along the boundary makes use of the unitary connections on $E_{1}$ and $E_{2}$. The boundary lagrangian is manifestly gauge-invariant. If we set $F=G=0$, we get the usual pathintegral representation of the parallel transport operator in the bundle $E_{1} \oplus E_{2}^{*}$ [16]. For non-zero $F$ or $G$ we get a deformation of the usual boundary condition. In the special case $G=0$ we get the boundary lagrangian used in ref. [16].

We postulate the following supersymmetry transformations for $\gamma$ :

$$
\delta \gamma=i \epsilon \bar{F}-i \bar{\epsilon} G .
$$

Here $\epsilon$ and $\bar{\epsilon}$ are regarded as independent complex Grassmann variables. We also note that $F$ and $G$ transform as follows:

$$
\begin{aligned}
& \delta F=\epsilon \psi^{i} \partial_{i} F \\
& \delta G=\epsilon \psi^{i} \partial_{i} G
\end{aligned}
$$

BRST transformations are obtained by setting $\epsilon=0$. One can check that the BRST variation of the boundary lagrangian is given, up to a total derivative, by

$$
\delta \mathcal{L}_{b}=-\frac{1}{2} \bar{\epsilon} \psi^{i} \partial_{i}(F G) .
$$

On the other hand, the BRST variation of the bulk action is a boundary term given by

$$
\delta S_{0}=\int \mathrm{d} \tau \bar{\epsilon}\left[g_{i \bar{j}} \partial_{0} \phi^{i}\left(\psi_{-}^{\bar{j}}-\psi_{+}^{\bar{j}}\right)+g_{i \bar{j}} \partial_{1} \phi^{i}\left(\psi_{-}^{\bar{j}}+\psi_{+}^{\bar{j}}\right)+\frac{i}{2}\left(\psi_{-}^{i}+\psi_{+}^{i}\right) \partial_{i} W\right] .
$$

The first two terms in the bulk variation are standard and vanish when the standard Neumann boundary conditions are imposed on $\phi^{i}$ and $\psi_{ \pm}^{i}$. The last term is the Warner 
term [33]. Obviously, in order for the variation of the boundary lagrangian to cancel the Warner term, we need to require

$$
F(\phi) G(\phi)=i(W(\phi)+\text { const }) .
$$

One can get rid of the factor $i$ by redefining $G \rightarrow i G$. This implies that instead of an ordinary complex of holomorphic vector bundles we are dealing with an object of $C\left(X, W, W_{0}\right)$.

It is straightforward to generalize the construction to the higher-rank case. The fermion $\gamma$ still takes values in $\operatorname{Hom}\left(E_{2}, E_{1}\right)$, which means that it is a matrix of size $\operatorname{rank}\left(E_{1}\right) \times$ $\operatorname{rank}\left(E_{2}\right)$. In order for the path-integral over $\gamma(\tau)$ to reproduce a path-ordered exponential in the representation of the gauge group of dimension $\operatorname{rank}\left(E_{1}\right)+\operatorname{rank}\left(E_{2}\right)$, one needs to insert a projector onto the sector where the total fermion number (including the boundary contribution from $\gamma$ ) is equal to 0 or 1 [20, 21, 19]. The rest of the argument is unchanged. The conditions of BRST-invariance now read

$$
F G=i(W+\text { const }), \quad G F=i(W+\text { const }) .
$$

The two conditions arise by requiring that the BRST variation of the bulk term be cancelled on both boundaries of the world-sheet. By taking the trace of these two equations and comparing them, one infers that the ranks of $E_{1}$ and $E_{2}$ are in fact the same, and the constant terms in the equations are also the same. It follows that any object of $C\left(X, W, W_{0}\right)$ is a B-brane.

One can also check that the total BRST charge is nilpotent. Indeed, it is easy to see that the square of the bulk contribution to the BRST charge is equal to

$$
Q_{0}^{2}=\frac{1}{2}\left\{Q_{0}, Q_{0}\right\}=-\left.i W\right|_{\partial \Sigma}
$$

On the other hand, the boundary supercharge coming from one of the two boundaries is given by

$$
Q_{b}=-i F \gamma+i G \bar{\gamma}
$$

Canonical quantization yields

$$
\{\gamma, \bar{\gamma}\}=1
$$

and therefore

$$
Q_{b}^{2}=F G
$$

It is also easy to check that $Q_{b}$ and $Q_{0}$ anti-commute (the holomorphicity of $F$ and $G$ is important here.) Hence the sum of $Q_{0}$ and the two boundary supercharges is nilpotent.

\subsection{Checking Kontsevich's proposal}

We start with the case $X=\mathbb{C}, W=z^{2}$, where there is only a D0-brane to worry about. In the absence of the superpotential, D0-brane on $\mathbb{C}$ is associated with the structure sheaf of a point, which has a two-term projective resolution

$$
\mathcal{O} \stackrel{z}{\longrightarrow} \mathcal{O} \text {. }
$$


If we pass to $\mathbb{Z}_{2}$-graded complexes, we obtain the following dg-module:

$$
\mathcal{O} \oplus \mathcal{O} \stackrel{z \sigma_{-}}{\longrightarrow} \mathcal{O} \oplus \mathcal{O}
$$

where

$$
\sigma_{-}=\left(\begin{array}{ll}
0 & 0 \\
1 & 0
\end{array}\right),
$$

and it is understood that the module $\mathcal{O} \oplus \mathcal{O}$ has the obvious grading. If we turn on the superpotential, we need to deform the differential so that its square be equal to $W=z^{2}$. It is clear how to do this: simply consider the object

$$
\mathcal{O} \oplus \mathcal{O} \stackrel{z \sigma_{1}}{\longrightarrow} \mathcal{O} \oplus \mathcal{O}
$$

where

$$
\sigma_{1}=\left(\begin{array}{ll}
0 & 1 \\
1 & 0
\end{array}\right) .
$$

This is our candidate object for the D0-brane at $z=0$. As a check, let us compute its endomorphism algebra, following Kontsevich's prescription. In the category $C\left(X, W, W_{0}\right)$, the algebra of endomorphisms is

$$
\operatorname{End}_{\mathcal{O}}(\mathcal{O}) \otimes_{\mathbb{C}} \operatorname{Mat}(2, \mathbb{C}) \simeq \operatorname{Mat}(2, \mathbb{C}[z])
$$

The $\mathbb{Z}_{2}$-grading is the natural grading on $2 \times 2$ matrices (diagonal elements are even, off-diagonal elements are odd). The differential acts on this graded vector space as follows:

$$
D:\left(\begin{array}{ll}
A & B \\
C & D
\end{array}\right) \mapsto\left(\begin{array}{cc}
(B+C) z & (A-D) z \\
-(A-D) z & (B+C) z
\end{array}\right) .
$$

Here $A, B, C, D$ are elements of $\operatorname{End}_{\mathcal{O}}(\mathcal{O})$, i.e. simply polynomials in $z$. Computing $H^{*}$, we find that this abelian group is isomorphic to the group of complex matrices of the form

$$
\left(\begin{array}{cc}
\xi & \eta \\
-\eta & \xi
\end{array}\right)
$$

Multiplicative structure is given by matrix multiplication. Clearly, this algebra is generated over $\mathbb{C}$ by the identity and an odd matrix

$$
\sigma_{2}=\left(\begin{array}{cc}
0 & -i \\
i & 0
\end{array}\right)
$$

which squares to identity. This agrees with the endomorphism algebra of the D0-brane in the LG model $W=z^{2}$ [16].

Now let us discuss B-branes in the LG model $W=x y$. Using the same reasoning as above, it is easy to guess that the D2-brane given by the equation $x=0$ should correspond to the object

$$
\mathcal{O} \oplus \mathcal{O} \longrightarrow \mathcal{O} \oplus \mathcal{O}
$$


where the map is defined as

$$
\left(\begin{array}{ll}
0 & y \\
x & 0
\end{array}\right)
$$

Similarly, the D2-brane given by the equation $y=0$ should correspond to

$$
\mathcal{O} \oplus \mathcal{O} \longrightarrow \mathcal{O} \oplus \mathcal{O}
$$

with the twisted differential

$$
\left(\begin{array}{ll}
0 & x \\
y & 0
\end{array}\right)
$$

The group of endomorphisms of the former object in the category $C\left(X, W, W_{0}\right)$ is

$$
\operatorname{End}_{\mathcal{O}}(\mathcal{O}) \otimes \operatorname{Mat}(2, \mathbb{C}) \simeq \operatorname{Mat}(2, \mathbb{C}[x, y]),
$$

with the differential which acts as follows:

$$
\left(\begin{array}{ll}
A & B \\
C & D
\end{array}\right) \mapsto\left(\begin{array}{cc}
B x+C y & (A-D) y \\
-(A-D) x & B x+C y
\end{array}\right) .
$$

The homology is readily computed; the result is that it is spanned by the identity matrix. Thus the algebra of endomorphisms in the derived category is isomorphic to $\mathbb{C}$. This agrees with the computation in section 5 . Of course, for the other D2-brane we get the same result. Finally, in order to compute morphisms between the two D2-branes, we note that one is a shift of the other. ${ }^{6}$ Thus the space of morphisms is the space of endomorphisms with gradings reversed, i.e. it is spanned by the identity matrix regarded as odd. Composing two such odd morphisms going in the opposite directions we get the identity endomorphism. This agrees with the computations in section 5 and explains why we declared the space of morphisms between two different D2-branes to be odd.

Now let us discuss the D0-brane. Consider the direct sum of objects corresponding to D2-branes with equations $x=0$ and $y=0$. It is easy to see that its algebra of endomorphism is the Clifford algebra $\mathrm{Cl}(2, \mathbb{C})$, so we propose that this object corresponds to the D0-brane. It is easy to check that morphisms to and from other objects agree with our computations in section 5 .

Finally, we propose an object of $H^{*}\left(C\left(X, W, W_{0}\right)\right)$ corresponding to the D0-brane in the free massive LG model with $n$ fields. If we bring the superpotential to the standard form eq. (6.1), then we can simply tensor $n$ copies of the object eq. (7.1). Consequently, the endomorphism algebra will also be the graded tensor product of $n$ copies of $\mathrm{Cl}(1, \mathbb{C})$, which is isomorphic to $\mathrm{Cl}(n, \mathbb{C})$. More invariantly, let $V=X$ be the complex vector space whose coordinates we denoted by $z_{i}$, let $e_{i}, i=1, \ldots, n$, be the corresponding basis in $V$, let $e_{i}^{*}, i=1, \ldots, n$ be the dual basis in $V^{*}$, and let $Q \in \operatorname{Sym}^{2}\left(V^{*}\right)$ be the hessian of $W$. We start with the $\mathbb{Z}_{2}$-graded version of the Koszul resolution of the point at the origin:

$$
\Omega^{\text {even }} \rightarrow \Omega^{\text {odd }}
$$

\footnotetext{
${ }^{6}$ In physical terms, this means that the D2-brane with the equation $x=0$ is isomorphic to the anti-brane for the D2-brane with the equation $y=0$.
} 
where $\Omega^{i}=\wedge^{i} V \otimes \mathcal{O}_{V}$, and the differential is induced by the wedge product with $z_{i} e_{i}$ (we use Einstein's convention of summing over repeating indices). Now we modify the differential so that its square be $W$ instead of zero. The obvious guess is

$$
d=z_{i}\left(e_{i}+\frac{1}{2} Q_{i j} i\left(e_{j}^{*}\right)\right)
$$

where $i(u), u \in V^{*}$, denotes the interior product with an element of $V^{*}$. Using the identity

$$
\left\{e_{i}, i\left(e_{j}^{*}\right)\right\}=\delta_{i j}
$$

one can easily check that $d^{2}=W$. Note that $d$ is essentially the Fourier transform of the Dirac operator, if we identify $\Omega^{\text {even }}$ and $\Omega^{\text {odd }}$ with spinor bundles.

\subsection{B-branes, Clifford modules, and Koszul duality ${ }^{7}$}

We regard the above computations as a convincing check of Kontsevich's proposal for massive LG models. In this subsection, we would like to address the following three questions. First, how do we match B-branes with objects in the category $H^{*}\left(C\left(X, W, W_{0}\right)\right)$ if $n>2$ ? (As explained in section 3, it is sufficient to consider the case $X=\mathbb{C}^{n}, W$ quadratic nondegenerate, and $W_{0}=0$.) Second, is there an efficient method to compute morphisms in the category $H^{*}\left(C\left(X, W, W_{0}\right)\right)$ ? Third, assuming the validity of Kontsevich's proposal, what do we learn about B-branes in massive LG models? That is, is there a simpler way to describe $H^{*}\left(C\left(X, W, W_{0}\right)\right)$ ?

To answer these questions, we will define a functor from the category of B-branes to the category of finite-dimensional $\mathbb{Z}_{2}$-graded modules over the Clifford algebra $\mathrm{Cl}(n, \mathbb{C})$. Let us denote this category $\operatorname{CLMOD}(n)$. (As usual, we allow both even and odd morphisms; thus $\operatorname{CLMOD}(n)$ is a $\mathbb{Z}_{2}$-graded category.) Since we set $X=\mathbb{C}^{n}, W_{0}=0$, and $W$ is quadratic and non-degenerate, the category $H^{*}\left(C\left(X, W, W_{0}\right)\right)$ really depends only on $n$; we will denote this category $\mathrm{K}(n)$ for short. There is a further functor from $\operatorname{CLMOD}(n)$ to $\mathrm{K}(n)$. Composing these two functors gives a way to associate objects of $\mathrm{K}(n)$ to Bbranes. In fact, as explained below, the second functor is an equivalence of categories which implies that we can calculate morphisms in $\operatorname{CLMOD}(n)$ instead of $\mathrm{K}(n)$. This equivalence is a cousin of the much-studied Koszul duality for quadratic algebras (see below). The structure of $\operatorname{CLMOD}(n)$ is quite simple: any object is a direct sum of irreducible objects (spinor modules), and there is one or two non-isomorphic irreducible objects, depending on whether $n$ is odd or even. Thus we have a completely explicit description of $\operatorname{CLMOD}(n)$, and therefore, by Koszul duality, of K $(n)$. Assuming the validity of Kontsevich's conjecture, this amounts to a solution of topological open string theory for any massive LG model.

One can associate a $\mathbb{Z}_{2}$-graded Clifford module to a B-brane as follows. For any Bbrane $Y$, consider the graded vector space $M(Y)=\operatorname{Mor}(D 0, Y)$. Since $\operatorname{Mor}(D 0, D 0)$ is isomorphic to $\mathrm{Cl}(n, \mathbb{C})$ as a graded algebra, $M(\cdot)$ is a functor from the category of B-branes to the category of left $\mathbb{Z}_{2}$-graded modules over $\mathrm{Cl}(n, \mathbb{C})$. Since spaces of open strings are

\footnotetext{
${ }^{7}$ The content of this subsection was explained to us by Alexander Polishchuk.
} 
expected to be finite-dimensional, $M(Y)$ is expected to be a finite-dimensional vector space. Thus $M(\cdot)$ is a graded functor from the graded category of B-branes to $\operatorname{CLMOD}(n)$.

The results of section 6 (see also the appendix imply that the functor $M(\cdot)$ maps the maximal linear B-brane to an irreducible Clifford module; for even $n$ maximal isotropic subspaces which belong to different irreducible families are mapped to non-isomorphic Clifford modules related by parity reversal. The D0-brane is mapped to the free module of rank one. A linear B-brane of complex dimension $\ell<[n / 2]$ is mapped to a module which is a direct sum of $2^{[n / 2]-\ell}$ irreducible modules.

Next we would like to explain why $\operatorname{CLMOD}(n)$ is equivalent to $\mathrm{K}(n)$. The relation between these two rather different-looking categories is a generalization of the so-called Koszul duality for quadratic algebras [34, 35, 36]. Any serious attempt to discuss Koszul duality would take us out of our depth, so we will just make a few remarks which may help to orient the reader who would like to study these questions deeper.

Classical Koszul duality applies to quadratic algebras, i.e. $\mathbb{Z}$-graded algebras generated by degree-1 elements, such that all relations between generators are homogeneous quadratic. The basic example of a dual pair is the pair $\left(S^{*}\left(V^{*}\right), \wedge^{*} V\right)$, where $S^{*}\left(V^{*}\right)$ is the symmetric algebra of a finite-dimensional vector space $V^{*}$, and $\wedge^{*} V$ is the exterior algebra of the dual vector space. The statement of Koszul duality is that their derived categories of finitely-generated $\mathbb{Z}$-graded modules are equivalent.

There is a generalization of Koszul duality to the case where the relations are nonhomogeneous quadratic [34, 37, 38]. But the dual object in this case is not a graded algebra, but a quadratic CDG algebra. A CDG algebra is a triple $(A, d, f)$, where $A$ is a graded algebra, $d$ is a degree- 1 derivation, and $f$ is a degree- 2 element $f$ such that $d^{2} a=[f, a]$ for any $a \in A$. CDG means "curved differential graded"; another name for a CDG algebra is a "Q-algebra" [39. A module over a CDG algebra $(A, d, f)$ is a graded module $M$ over $A$ equipped with a degree-1 derivation $d_{M}$ such that $d_{M}^{2} m=f \cdot m$ for any $m \in M$.

What we need is a $\mathbb{Z}_{2}$-graded version of non-homogeneous Koszul duality. Indeed, on one hand, the Clifford algebra is a $\mathbb{Z}_{2}$-graded quadratic algebra, while on the other hand, the category $C\left(X, W, W_{0}\right)$ can be regarded as a category of modules over a certain $\mathbb{Z}_{2}$-graded CDG algebra. This CDG algebra is purely even and isomorphic to $\mathcal{O}_{X}$ as an algebra. The derivation $d$ is identically zero, but the even element $f$ is not: it is given by $W$. The category $\mathrm{K}(n)$ can be regarded as the derived category of the category of finitely generated CDG modules over the CDG algebra $\left(\mathcal{O}_{X}, 0, W\right)$. This CDG algebra is Koszul-dual to the Clifford algebra in the sense of refs. [37, 38], and we expect that the corresponding derived categories of modules are equivalent. More precisely, we expect that the derived category of finite-dimensional $\mathbb{Z}_{2}$-graded Clifford modules is equivalent to the derived category of finitely-generated modules over the CDG algebra $\left(\mathcal{O}_{X}, 0, W\right)$.

Since the Clifford algebra can be regarded as a deformation of the exterior algebra, and the CDG algebra $\left(\mathcal{O}_{X}, 0, W\right)$ is a deformation of the polynomial algebra, this claim looks like a generalization of the classic result of ref. [35]. In fact, the deformed duality is in some sense simpler than the classic one, since the category $\operatorname{CLMOD}(n)$ is semi-simple and "deriving" it is a trivial operation (gives us back the same category). It is also more useful: while the classic duality of ref. [35] reduced the problem of classifying coherent sheaves on 
$\mathbb{C P}^{n}$ to a very difficult problem in linear algebra, the deformed duality reduces the problem of classifying B-branes in the free massive LG model to a very simple problem in linear algebra (classification of finite-dimensional graded modules over a Clifford algebra.)

Let us describe the functors which establish the equivalence of $\mathrm{K}(n)$ and $\operatorname{CLMOD}(n)$. The first one, from $\mathrm{K}(n)$ to $\operatorname{CLMOD}(n)$, is obvious: it takes an object $Y$ of $\mathrm{K}(n)$ to $\operatorname{Mor}\left(Y_{0}, Y\right)$, where $Y_{0}$ is the object of $\mathrm{K}(n)$ described in the last paragraph of subsection 7.3 . The mapping of morphisms is the obvious one.

To define the functor acting in the opposite direction, let us consider for any object $M$ of $\operatorname{CLMOD}(n)$ the vector space

$$
N=M \otimes_{\mathbb{C}} \mathcal{O}_{X}
$$

where $\mathcal{O}_{X}$ is simply the algebra of polynomial functions on $\mathbb{C}^{n}$. Since $M$ is $\mathbb{Z}_{2}$-graded, this vector space is also $\mathbb{Z}_{2}$-graded. It is also an $\mathcal{O}_{X}$ module, for obvious reasons. It remains to define the twisted differential $d_{N}$, i.e. an odd endomorphism of $N$ which squares to $W$. Let $V$ be the vector space which appears in the definition of the Clifford algebra; we will also identify the target space $X$ of the LG model with $V$. The twisted differential will be

$$
d_{N}: m \otimes f \mapsto \sum_{i}\left(e_{i} \cdot m\right) \otimes z_{i} f, \quad \forall m \in M, \quad \forall f \in \mathcal{O}_{X}
$$

where $e_{i}, i=1, \ldots, n$, is a basis in $V, z_{i}$ are the corresponding linear coordinates, and the dot denotes the Clifford algebra action. It is easy to check that $d_{N}$ is odd, and that $d_{N}^{2}=W$. Thus we defined a map which sends an object of $\operatorname{CLMOD}(n)$ to an object of $\mathrm{K}(n)$. The mapping of morphisms is the obvious one: if $\alpha$ is a morphism of Clifford modules $M$ and $M^{\prime}$, then the corresponding element of $\operatorname{Hom}_{\mathcal{O}_{X}}\left(N, N^{\prime}\right)$ is $\alpha \otimes 1$. It is easy to check that $\alpha \otimes 1$ is closed, and thus is a well-defined morphism in the category $\mathrm{K}(n)$.

The claim is that compositions of these two functors in any order are isomorphic to identity functors. We will not try to prove this claim here, but to make it more plausible note that the mapping of objects is given by essentially the same formulas as in the classic case [35].

\section{Application: the category of A-branes for some Fano varieties}

\subsection{A-branes on $\mathbb{C P}^{2}$}

The mirror of the the nonlinear sigma model with target $\mathbb{C P}^{2}$ is the affine $A_{2}$ Toda model [5]. The affine $A_{2}$ Toda model is an $\mathcal{N}=2$ Landau-Ginzburg theory of two chiral superfields $x$ and $y$ taking values in $\mathbb{C}^{*}$ and a rational superpotential

$$
W(x, y)=x+y+\frac{1}{x y} .
$$

We can test the Homological Mirror Symmetry conjecture by comparing the Fukaya category of $\mathbb{C P}^{2}$ with the category of B-branes in the Toda model.

As discussed above, every B-brane in the Toda model lies on some holomorphic curve $W=W_{0}$. In addition, in order for open strings to have a supersymmetric ground state, we 


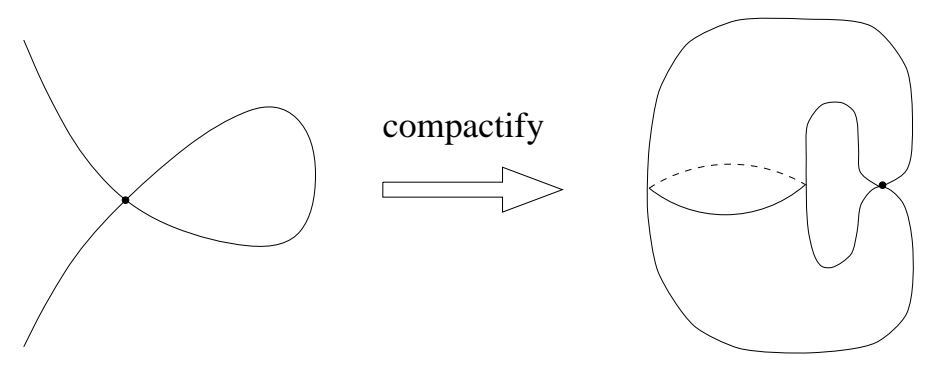

Figure 2.

require this curve to pass through a critical point of $W$. In the $A_{2}$ theory there are three distinct critical points:

$$
x=y=a_{k}=e^{2 k \pi i / 3}, \quad k=0,1,2 .
$$

The values of $W$ corresponding to these critical points are pairwise distinct: $W_{k}=3 a_{k}$. There is an obvious $\mathbb{Z}_{3}$ symmetry which permutes the critical points. This implies that the categories $H^{*}\left(C\left(X, W, W_{k}\right)\right)$ are all equivalent. From now on we will focus on one of them, say, the one corresponding to $k=0$. All B-branes associated to this critical point must be complex submanifolds of the holomorphic curve in $\mathbb{C}^{*} \times \mathbb{C}^{*}$ given by

$$
x+y+\frac{1}{x y}-3=0 .
$$

This curve is a singular cubic with a single node (see figure ??). Thus the category of B-branes is a full sub-category of the category of B-branes in the LG model $W=x y$. We have seen that the latter is equivalent to the category $\operatorname{CLMOD}(2)$.

It remains to understand which objects in the latter category correspond to B-branes. Clearly, the D0-brane sitting at the critical point $(1,1)$ is a valid B-brane. As for D2-branes, they must be (desingularizations of the) irreducible components of the curve eq. (8.1). But it is easy to see that the singular cubic is irreducible. Thus there is only one D2-brane of type B: the one which corresponds to the structure sheaf of the desingularized cubic. The corresponding object in the "local" category associated to the critical point is the direct sum of the D2-brane $x=0$ and the D2-brane $y=0$. This direct sum is isomorphic to the D0-brane (see section (5)). We conclude that the basic B-brane in the Toda model is the D0-brane, all other branes being direct sums of several copies of the D0-brane. The endomorphism algebra of the D0-brane is isomorphic to $\mathrm{Cl}(2, \mathbb{C})$. We see that the category of B-branes in this case is strictly smaller than the "local" category $\mathrm{C}_{\text {tot }}$, which is equivalent to $\operatorname{CLMOD}(2)$.

As discussed in section 3, since the D0-brane looks like a composite of two D2-branes, one can formally add these missing D2-branes to the category of B-branes for the Toda model. The enlarged category is equivalent to the category $\operatorname{CLMOD}(2)$.

Now let us interpret these results from the point of view of Homological Mirror Symmetry. The mirror of the D0-brane has been identified in ref. 16 using the dualization argument of ref. [5]. The mirror is a certain lagrangian 2-torus in $\mathbb{C P}^{2}$ equipped with a rank 
one trivial vector bundle and a certain flat connection. Let us be more specific. Consider the unit 5 -sphere in $\mathbb{C}^{3}$, i.e. a hypersurface defined by the equation

$$
\left|z_{1}\right|^{2}+\left|z_{2}\right|^{2}+\left|z_{3}\right|^{2}=1
$$

The quotient of this 5 -sphere by a free $\mathbb{S}^{1}$ action

$$
z_{i} \rightarrow e^{2 \pi i \alpha} z_{i}, \quad i=1,2,3, \quad \alpha \in \frac{\mathbb{R}}{\mathbb{Z}}
$$

is diffeomorphic to $\mathbb{C P}^{2}$. In fact, the standard symplectic form on $\mathbb{C P}^{2}$ is obtained by restricting to $\mathbb{S}^{5}$ the standard Kähler form on $\mathbb{C}^{3}$ and then pushing it down to the quotient. Now consider a 3 -torus in $\mathbb{C}^{3}$ defined by the equations

$$
\left|z_{1}\right|^{2}=\left|z_{2}\right|^{2}=\left|z_{3}\right|^{2}=\frac{1}{3}
$$

It is contained in the 5 -sphere and invariant with respect to the $\mathbb{S}^{1}$ action. Hence by passing to the quotient, we obtain a 2 -torus embedded in $\mathbb{C P}^{2}$. It is trivial to check that this 2-torus is lagrangian with respect to the standard symplectic form on $\mathbb{C P}^{2}$. The flat connection can be specified by its monodromy representation. Let $\gamma_{1}$ and $\gamma_{2}$ be the loops on the 3-torus (8.2) defined by

$$
\gamma_{1}: t \mapsto\left\{\frac{1}{\sqrt{3}} e^{2 \pi i t}, \frac{1}{\sqrt{3}}, \frac{1}{\sqrt{3}}\right\}, \quad \gamma_{2}: t \mapsto\left\{\frac{1}{\sqrt{3}}, \frac{1}{\sqrt{3}} e^{2 \pi i t}, \frac{1}{\sqrt{3}}\right\} .
$$

Their images under the quotient map generate the fundamental group of our lagrangian 2-torus. According to ref. [16], the mirror of the D0-brane sitting at the point $\left(a_{k}, a_{k}\right)$, $k=0,1,2$, corresponds to the monodromy representation which maps both generators to $e^{2 \pi i k / 3}$. In particular, the D0-brane which sits at the point $(1,1)$ is mirror to the trivial flat connection on the lagrangian 2-torus.

As a simple check of this claim, note that the algebra of endomorphisms of a D0-brane in the model $W=x y$ has Euler characteristic zero. In the mirror picture, the corresponding object is the Euler characteristic of the Floer complex, which coincides with the classical Euler characteristic of the 2-torus. Thus the Euler characteristics match. It would be nice to compute the Floer homology groups as well and to check that they agree with the predictions of mirror symmetry. Namely, we expect that

1. the Floer homology of the lagrangian 2-torus equipped with a rank-one flat connection is non-vanishing only for the three special flat connections defined above;

2. for these choices of the flat connection, the Floer homology is isomorphic to the classical cohomology of the torus as a $\mathbb{Z}_{2}$-graded vector space;

3. as a $\mathbb{Z}_{2}$-graded algebra, the Floer homology is isomorphic to the Clifford algebra with two generators, i.e. it is a quantum deformation of the classical cohomology ring;

4. Floer homology groups which compute morphisms between different flat connections of rank one vanish. 
It was argued above that we can formally add D2-branes to the category of B-branes. It is reasonable to ask if this procedure is consistent with or perhaps even forced on us by Homological Mirror Symmetry. To answer this question we need to identify the mirrors of the added D2-branes. There are two such D2-branes for each critical level set. For each of them the Euler characteristic of the endomorphism algebra is 1. If we assume that the mirror of a D2-brane is a lagrangian submanifold, then it must be homeomorphic to a real projective plane $\mathbb{R P}^{2}$. But since $\mathbb{R} \mathbb{P}^{2}$ is not orientable, it is not an admissible object of the Fukaya category (one needs orientability in order to define $\mathbb{Z}_{2}$-graded Maslov index and $\mathbb{Z}_{2}$-grading on the Floer complex). We conclude that the mirrors of the added D2-brane cannot be lagrangian submanifolds, and therefore Homological Mirror Symmetry does not force us to include them on the B-side.

On the other other hand, if we added D2-branes on the B-side, we can maintain Homological Mirror Symmetry by adding certain objects on the A-side. In other words, we would like to regard the lagrangian 2-torus with a trivial flat connection, which is mirror to the D0-brane, as a direct sum of two irreducible objects, which are mirror to the D2branes. But since there are no such objects in the Fukaya category, we simply add these direct summands "by hand."

Let us clarify what we mean by adding direct summands "by hand." Let $E$ be an object of an additive category C. A projector is an element of $\operatorname{End}(E)$ which satisfies $e \circ e=e$. Given any projector, we would like to have the corresponding direct summand, i.e. an object $R$ and a pair of morphisms $i: R \rightarrow E$ and $r: E \rightarrow R$ such that $r \circ i=i d_{R}$ and $i \circ r=e$. If $R$ does not exist for all projectors and for all $E$, then we look for the smallest additive category which contains $C$ as a full subcategory and in which every projector has a direct summand.

To summarize, to maintain Homological Mirror Symmetry, we must either add formal direct summands on both A and B sides, or on neither side.

\subsection{A-branes on $\mathbb{C P}^{1} \times \mathbb{C P}^{1}$}

The mirror in this case is the LG model with target $\mathbb{C}^{*} \times \mathbb{C}^{*}$ and the superpotential

$$
W(x, y)=x+\frac{\mu}{x}+y+\frac{\nu}{y} .
$$

Here $\mu$ and $\nu$ are nonzero complex numbers whose logarithms are mirror to the periods of the complexified Kähler form on the two $\mathbb{C P}^{1}$ 's. This superpotential has four nondegenerate critical points. For generic $\mu, \nu$ there are four critical level sets all of which look like a cubic with a node. Thus we are in exactly the same situation as in the previous subsection, and the only B-branes are D0-branes sitting at the critical points. Another way to see these D0-branes is to note that the LG model is a product of two LG models with the superpotential $W=x+\mu / x$. This model is mirror to $\mathbb{C P}^{1}$ and has been studied in ref. [16]. Its only B-branes are D0-branes sitting at the two critical points of the superpotential. Taking tensor products of pairs of such B-branes gives us four D0-branes discussed above.

The mirror of each D0-brane is a lagrangian 2-torus with some flat connection. Indeed, the mirror of a D0-brane in the model $W=x+\mu / x$ is the equatorial circle on $\mathbb{C P}^{1}$ [16], 
therefore the mirror of a D0-brane in the product model is the product of two equatorial circles. The monodromy around the two generators of the fundamental group is $( \pm 1, \pm 1)$.

For $\mu=\nu$ something special happens both on the A and B sides. On the A side, we get a new lagrangian submanifold which is homeomorphic to a 2 -sphere. To see this, let $z$ and $w$ be coordinates on the standard affine patches on the two $\mathbb{C P}^{1}$ 's. Consider the "antidiagonal" 2 -sphere given by $z=\bar{w}$. Let $\omega$ be the Fubini-Study form on $\mathbb{C P}^{1}, \pi_{i}, i=1,2$, be the projection maps from $\mathbb{C P}^{1} \times \mathbb{C P}^{1}$ to the two factors, and $a_{1}, a_{2}$ be complex numbers. It is trivial to check the restriction of $a_{1} \pi_{1}^{*} \omega+a_{2} \pi_{2}^{*} \omega$ to the "anti-diagonal" 2 -sphere vanishes if and only if $a_{1}=a_{2}$. Thus the 2-sphere is lagrangian if and only if $\mu=\nu$.

On the $\mathrm{B}$ side, the critical level set $W=0$ now contains two critical points. The equation of this critical level set

$$
(x+y)(x y+\mu)=0
$$

shows that it is reducible. The irreducible components are a line and a non-singular quadric which intersect transversally at two points; these are the two critical points mentioned above. We have two irreducible D2-branes of type $\mathrm{B}$ corresponding to the two irreducible components of the critical level set. It is easy to see that one is isomorphic to the shift of the other, while their sum is isomorphic to the sum of two D0-branes sitting at the two critical points.

Note that this is another example where the category of B-branes is strictly smaller than the sum of "local" categories associated to critical points. This happens because all D2-branes pass through both critical points in the set $W=0$. Thus a single D0-brane sitting at a critical point is irreducible. Of course, if we only look at the infinitesimal neighborhood of one of the critical points, then we are in the same situation as in the model $W=x y$, and the D0-brane appears to be composite. If desired, we can enlarge the category of B-branes by adding all formal direct summands. Then it will become equivalent to the sum of categories attached to the two critical points (each of which is equivalent to CLMOD(2)), and each D0-brane will be the sum of two irreducible objects.

Now let us match the objects on A and B sides. D0-branes correspond to "equatorial" lagrangian tori, as before. The mirror of a D2-brane must be a lagrangian 2-sphere. Indeed, each D2-brane passes through two critical points, each of which contributes 1 to the Euler characteristic of the endomorphism algebra. An obvious conjecture is that the two D2branes are mirror to the lagrangian 2-sphere discussed above and its shift (i.e. orientationreversal). If this is true, then the sum of the lagrangian 2-sphere and its shift must be isomorphic (in the Fukaya category) to the sum of two "equatorial" lagrangian tori with monodromies $(1,-1)$ and $(-1,1)$. It would be interesting to check this by computing the Floer homology between all the objects involved.

\section{Comments and outlook}

In this paper we have described the category of B-branes for the free massive LG model with $n$ chiral fields. We also argued that this allows one to determine the category of B-branes for an arbitrary massive LG model. The most striking feature of our results is 
their simplicity. For example, if we consider the free massive LG model, there is a multiparameter family of maximal isotropic subspaces of the quadric $W=0$, but they are all isomorphic as objects of the category of B-branes (up to a shift). Moreover, B-branes of lower dimension, including the D0-brane, are isomorphic to direct sums of B-branes of maximal dimension. These rather counter-intuitive observations solve the problem of computing tree-level topological open string correlators in these models.

It is interesting to compare our results with those of refs. [22, 23], where a general framework for classifying D-branes in 2d Topological Field Theories has been proposed. In our case, the $2 \mathrm{~d}$ TFT in the bulk is rather trivial: it is isomorphic, as a Frobenius algebra, to $\mathbb{C}$ with its unique Frobenius structure. The theory of ref. [22] (generalized to the $\mathbb{Z}_{2^{-}}$ graded case) tells us that the algebra of open strings connecting a brane with itself must be simple. We saw that in our case endomorphism algebras of B-branes are all isomorphic to Clifford algebras, and these are indeed simple (as $\mathbb{Z}_{2}$-graded algebras). However, unlike in the purely bosonic case, in the $\mathbb{Z}_{2}$-graded case not every two simple finite-dimensional algebras are Morita equivalent. In fact, there are two Morita-equivalence classes of such algebras, represented by $\mathbb{C}$ and $\mathrm{Cl}(1, \mathbb{C})$. A Clifford algebra with $k$ generators is Moritaequivalent to $\mathbb{C}$ or $\mathrm{Cl}(1, \mathbb{C})$ depending on whether $k$ is even or odd. We have seen that when the number of fields $n$ is even (resp. odd) only even (resp. odd) values of $k$ occur. This suggests that it is impossible to have a topological open string theory which includes D-branes of both kinds. Indeed, we have seen that all pairings between spaces of morphisms induced by 2-point correlators are either even or odd, depending on whether $n$ is even or odd. On the other hand, one of the basic axioms of topological open string theory is that all pairings must have the same parity [23].

This observation provides a simple counter-example to the belief that a $2 \mathrm{~d}$ TFT determines uniquely the associated category of topological boundary conditions. In fact, we can make a stronger statement. Given any $2 \mathrm{~d}$ SCFT representing a superstring background, we can tensor it with the topological LG model $W=z^{2}$. Since the latter theory is trivial, this does not change the closed string sector. But the open string sector does change: one has to tensor every "physical" D-brane with the D0-brane of the LG model, and this results in tensoring the open string spectrum of each D-brane with $\mathrm{Cl}(1, \mathbb{C})$. This is equivalent to the introduction of an odd Chan-Paton label. Thus we have two inequivalent open string theories for a given closed string theory.

This particular ambiguity is rather mild and can be easily eliminated. The difference between odd and even $n$ comes from the number of fermionic zero modes on a disk, or equivalently from the parity of the bilinear forms computed by the 2-point disk correlators. Thus to specify completely the open string theory we are dealing with, it is sufficient to fix the parity of all bilinear forms.

It would be interesting to extend the considerations of this paper to LG models which flow to non-trivial SCFTs in the infrared limit. For example, one could study LandauGinzburg realizations of $N=2$ minimal models. For these theories much information about B-branes is available from the boundary state formalism, and it would interesting to see if it is consistent with Kontsevich's proposal. By analogy with the massive case, one expects that the category of B-branes will be describable in terms of modules over 
the algebra of endomorphisms of a D0-brane. From the mathematical viewpoint, this algebra must be related by a Koszul-like duality to the CDG algebra which appears in Kontsevich's proposal. It appears that for $W$ of degree higher than two Koszul duality relates Kontsevich's CDG algebra to a finite-dimensional $A_{\infty}$-algebra [32]. B-branes should correspond to finite-dimensional $A_{\infty}$-modules over this $A_{\infty}$-algebra. In this way solving topological open string theory is reduced to a problem in linear algebra. Hopefully, the latter problem is manageable.

In the axiomatic approach of refs. 22, 22, topologically twisted $N=2$ minimal models correspond to non-semi-simple Frobenius algebras. It would be interesting to explore the uniqueness of the open string sector in such models.

\section{Acknowledgments}

We are deeply grateful to Maxim Kontsevich for sharing with us his ideas about B-branes in Landau-Ginzburg models, and to Alexander Polishchuk for pointing out the relevance of non-homogeneous Koszul duality. We also thank Kentaro Hori and Dmitri Orlov for reading a preliminary draft of the paper and making a number of valuable comments. The first author would like to thank Institut des Hautes Études Scientifiques for hospitality during the writing of this paper. This work was supported in part by the DOE grant DE-FG03-92-ER40701.

\section{A. Clifford algebras and modules}

In this appendix we collect some well-known facts about complex Clifford algebras and their modules. Let $V$ be a complex vector space of dimension $n$, and $Q$ be a non-degenerate symmetric bilinear form on $V$. Clifford algebra $\mathrm{Cl}(V, Q)$ has $V$ and the identity as its set of generators, and the following relations:

$$
v \cdot v^{\prime}+v^{\prime} \cdot v=Q\left(v, v^{\prime}\right)
$$

As a vector space, $\mathrm{Cl}(V, Q)$ is isomorphic to $\wedge^{*} V$ and therefore has dimension $2^{n}$. We can regard $\mathrm{Cl}(V, Q)$ either as an ordinary associative algebra, or as a $\mathbb{Z}_{2}$-graded algebra, such that all the generators are odd. In the latter case, the grading corresponds to the decomposition of $\wedge^{*} V$ into polyvectors of even and odd degree. Since the isomorphism class of $\mathrm{Cl}(V, Q)$ depends only on the dimension $n$ of $V$, we will also use the notation $\mathrm{Cl}(n, \mathbb{C})$ to denote this isomorphism class.

If $V_{1}$ and $V_{2}$ are complex vector spaces with non-degenerate bilinear forms $Q_{1}$ and $Q_{2}$, then

$$
\mathrm{Cl}\left(V_{1} \oplus V_{2}, Q_{1} \oplus Q_{2}\right)=\mathrm{Cl}\left(V_{1}, Q_{1}\right) \otimes \operatorname{Cl}\left(V_{2}, Q_{2}\right) .
$$

Here all Clifford algebras are regarded as $\mathbb{Z}_{2}$-graded algebras, and $\otimes$ denotes their $\mathbb{Z}_{2}$-graded tensor product. 
If $n$ is even, then $\mathrm{Cl}(V, Q)$ regarded as an ungraded algebra is isomorphic to the algebra of complex $2^{n / 2} \times 2^{n / 2}$ matrices, which we will denote $\operatorname{Mat}\left(2^{n / 2}, \mathbb{C}\right)$. If $n$ is odd, then $\mathrm{Cl}(V, Q)$ regarded as an ungraded algebra is isomorphic to $\operatorname{Mat}\left(2^{[n / 2]}, \mathbb{C}\right) \oplus \operatorname{Mat}\left(2^{[n / 2]}, \mathbb{C}\right)$. In particular, $\mathrm{Cl}(1, \mathbb{C})$ is isomorphic to $\mathbb{C} \oplus \mathbb{C}$. We see that $\mathrm{Cl}(V, Q)$ is a simple algebra only for even $n$. However, if we regard it as a $\mathbb{Z}_{2}$-graded algebra, then it is simple for all $n$.

Now let us discuss finite-dimensional modules over $\mathrm{Cl}(V, Q)$. The category of Clifford modules is semi-simple, i.e. every exact sequence splits. Thus every Clifford module is a direct sum of irreducible modules. The number and properties of irreducible modules depend on the parity of $n$, as well as whether we regard $\mathrm{Cl}(V, Q)$ as a $\mathbb{Z}_{2}$-graded algebra. If we neglect the grading, then for even $n$ we have a unique irreducible module $S$ of dimension $2^{n / 2}$. It is called the spinor module and can be constructed as follows. Pick a pair of subspaces $U, W$ of $V$ such that both $U$ and $W$ are isotropic with respect to $Q$, $U \cap W=0$, and $V=U \oplus W$. One can easily see that $Q$ gives a non-degenerate pairing between $U$ and $W$ and thus we may identify $W$ with $U^{*}$. Set $S=\wedge^{*} U$, and define the action of Clifford algebra on $S$ as follows: if $v=u \oplus w$, where $u \in U$ and $w \in W$, then for any $\lambda \in S$ we let

$$
(u \oplus w) \cdot \lambda=u \wedge \lambda+i_{w} \lambda .
$$

Here we used the identification of $W$ with $U^{*}$ mentioned above.

For odd $n$ Clifford algebra is a sum of two matrix algebras, and therefore there are two non-isomorphic irreducible modules of dimension $2^{[n / 2]}$ (two spinor modules). For example, for $n=1$ the algebra is generated by the identity and an odd element $\xi$ with a single relation $\xi^{2}=1$; the two irreducible modules are one-dimensional, with the action of $\xi$ given by \pm 1 . For general $n$ one can use the property eq. (A.1) to reduce the problem to the cases already considered.

If we regard $\mathrm{Cl}(V, Q)$ as a $\mathbb{Z}_{2}$-graded algebra, then we should look for $\mathbb{Z}_{2}$-graded irreducible modules. For even $n$ there are two inequivalent choices of grading on the spinor module related by parity reversal. Therefore we have two non-isomorphic irreducible spinor modules $S$ and $\bar{S}$. For odd $n$ the "minimal" $\mathbb{Z}_{2}$-graded module has dimension $2^{(n+1) / 2}$; as an ungraded module, it is isomorphic to the direct sum of two inequivalent irreducible ungraded modules. Furthermore, the choice of grading is unique up to isomorphism. We will denote this unique spinor module by $S$. For example, for $n=1 S \simeq \mathbb{C}^{2}$, and $\xi$ acts as any of the three Pauli matrices, say $\sigma_{1}$. Then the parity operator can be chosen to be $\sigma_{3}$.

To summarize, for even $n$ any $\mathbb{Z}_{2}$-graded Clifford module is a direct sum of several copies of two inequivalent spinor modules $S$ and $\bar{S}$. For odd $n$ the situation is the same, except that $S$ is isomorphic to $\bar{S}$. The dimension of the spinor module is given by $2^{[(n+1) / 2]}$ for any $n>0$.

In particular, $\mathrm{Cl}(V, Q)$ regarded as a left module over itself is a direct sum of $2^{[n / 2]}$ copies of spinor modules. For $n$ even half of them are $S$ 's, and the other half are $\bar{S}$ 's.

\section{References}

[1] M. Kontsevich, Homological algebra of mirror symmetry, alg-geom/9411018.

[2] E. Witten, Topological sigma models, Commun. Math. Phys. 118 (1988) 411. 
[3] E. Witten, Mirror manifolds and topological field theory, in Essays in mirror symmetry, S.T. Yau ed., International Press Co., Hong Kong 1992.

[4] C. Vafa, Topological Landau-Ginzburg models, Mod. Phys. Lett. A 6 (1991) 337.

[5] K. Hori and C. Vafa, Mirror symmetry, hep-th/0002222.

[6] J. Maldacena and L. Maoz, Strings on PP-waves and massive two dimensional field theories, J. High Energy Phys. 12 (2002) 046 hep-th/0207284.

[7] T. Eguchi and S.-K. Yang, N=2 superconformal models as topological field theories, Mod. Phys. Lett. A 5 (1990) 1693.

[8] M.R. Douglas, D-branes, categories and $N=1$ supersymmetry, J. Math. Phys. 42 (2001) 2818 hep-th/0011017.

[9] P.S. Aspinwall and A.E. Lawrence, Derived categories and zero-brane stability, J. High Energy Phys. 08 (2001) 004 hep-th/0104147.

[10] S. Katz and E. Sharpe, D-branes, open string vertex operators and ext groups, Adv. Theor. Math. Phys. 6 (2003) 979 hep-th/0208104.

[11] A. Kapustin and D. Orlov, Remarks on a-branes, mirror symmetry and the fukaya category, hep-th/0109098.

[12] K. Hori, A. Iqbal and C. Vafa, D-branes and mirror symmetry, hep-th/0005247.

[13] K. Hori et al., Mirror symmetry, Lectures at The Clay mathematical school on mirror symmetry, Spring 2000, to be published.

[14] S. Govindarajan, T. Jayaraman and T. Sarkar, Worldsheet approaches to D-branes on supersymmetric cycles, Nucl. Phys. B 580 (2000) 519 hep-th/9907131.

[15] S. Govindarajan and T. Jayaraman, On the Landau-Ginzburg description of boundary cfts and special lagrangian submanifolds, J. High Energy Phys. 07 (2000) 016 hep-th/0003242.

[16] K. Hori, Linear models of supersymmetric D-branes, hep-th/0012179.

[17] P. Seidel, Vanishing cycles and mutation, math.SG/0007115; More about vanishing cycles and mutation, math.SG/0010032.

[18] K. Hori, Mirror symmetry and quantum geometry, hep-th/0207068.

[19] S. Govindarajan and T. Jayaraman, Boundary fermions, coherent sheaves and D-branes on Calabi-Yau manifolds, Nucl. Phys. B 618 (2001) 50 hep-th/0104126.

[20] S. Hellerman and J. McGreevy, Linear sigma model toolshed for D-brane physics, J. High Energy Phys. 10 (2001) 002 hep-th/0104100.

[21] S. Hellerman, S. Kachru, A.E. Lawrence and J. McGreevy, Linear sigma models for open strings, J. High Energy Phys. 07 (2002) 002 hep-th/0109069.

[22] G. Moore and G. Segal, unpublished; See also lectures by G. Moore at http://online.itp.ucsb.edu/online/mp01.

[23] C.I. Lazaroiu, On the structure of open-closed topological field theory in two dimensions, Nucl. Phys. B 603 (2001) 497 hep-th/0010269.

[24] E. Abdalla and A. Lima-Santos, On some features of $\mathbb{C P}^{N-1}$ models with fermions, Phys. Rev. D 29 (1984) 1851. 
[25] R. Koberle and V. Kurak, Solitons in the supersymmetric $\mathbb{C P}^{N-1}$ model, Phys. Rev. D 36 (1987) 627.

[26] E. Abdalla, M. Forger and A. Lima Santos, Nonlocal charges for nonlinear sigma models on grassmann manifolds, Nucl. Phys. B 256 (1985) 145.

[27] U. Lindström and M. Zabzine, $N=2$ boundary conditions for non-linear sigma models and Landau-Ginzburg models, J. High Energy Phys. 02 (2003) 006 [hep-th/0209098].

[28] E. Witten, On the structure of the topological phase of two dimensional gravity, Nucl. Phys. B 340 (1990) 281.

[29] P. Griffiths and J. Harris, Principles of algebraic geometry, Wiley, New York 1994.

[30] A.I. Bondal and M.M. Kapranov, Framed triangulated categories, Math. Sb. 181 (1990) 669.

[31] S. I. Gelfand and Yu. I. Manin, Algebra V: homological algebra, A. I. Kostrikin and I. R. Shafarevich eds., Encyclopaedia of Mathematical Sciences 38, Springer 1994.

[32] M. Kontsevich, private communication.

[33] N.P. Warner, Supersymmetry in boundary integrable models, Nucl. Phys. B 450 (1995) 663 hep-th/9506064.

[34] S. B. Priddy, Koszul resolutions, Trans. Amer. Math. Soc. 152 (1970) 39.

[35] J.N. Bernstein, I.M. Gelfand, and S.I. Gelfand, Algebraic vector bundles on $\mathbb{P}^{n}$ and problems of linear algebra, Funktsional. Anal. i Prilozhen. 12 (1978) 66.

[36] A.A. Beilinson, V.A. Ginzburg, and V.V. Schechtman, Koszul duality, J. Geom. Phys. 5 (1988) 317.

[37] L. Positselski, Nonhomogeneous quadratic duality and curvature, Funktsional. Anal. i Prilozhen. 27 (1993) 57.

[38] A. Polishchuk and L. Positselski, Quadratic algebras, unpublished preprint.

[39] A. Schwarz, Noncommutative supergeometry and duality, Lett. Math. Phys. 50 (1999) 309 hep-th/9912212. 